\title{
Expedient synthesis of substituted
}

\section{(diphenylphosphinoylmethyl)benzenes.}

Sean P. Bew,* Rebecca A. Brimage, David L. Hughes, Laurent Legentil, Sunil V. Sharma and

Martin A. Wilson.

S.bew@uea.ac.uk

\section{General protocols}

All reactions requiring anhydrous conditions were conducted in flame-dried glass apparatus under an atmosphere of argon. Water refers to distilled water. All commercially available reagents were used as supplied.

The ${ }^{1} \mathrm{H}$-spectra were recorded in ppm and referenced to the residual $\mathrm{CHCl}_{3}$ signal located at $\delta$ 7.26. ${ }^{13} \mathrm{C}$-NMR spectra were recorded in ppm and referenced to the residual $\mathrm{CHCl}_{3}$ signal found at $\delta$ 77.00. Multiplicities in the NMR spectra are described as: $s=$ singlet, $d=$ doublet, $t=$ triplet, $q=$ quartet, $\mathrm{m}=$ multiplet, $\mathrm{br}=$ broad; coupling constants are reported in $\mathrm{Hz}$.

Page $1 \quad$ General protocols

Page 2 General protocols

Page 3 Procedure for the synthesis of $\mathbf{2}-\mathbf{3}$

Page $4 \quad$ Procedure for the synthesis of $\mathbf{4}$ - $\mathbf{5}$

Page $5 \quad$ Procedure for the synthesis of $\mathbf{7}$ and $\mathbf{8}$

Page $6 \quad$ Procedure for the synthesis of 9

Page $7 \quad$ Procedure for the synthesis of $\mathbf{1 0}$ and $\mathbf{1 1}$

Page 8 Procedure for the synthesis of $\mathbf{1 2}$

Page $9 \quad$ Procedure for the synthesis of $\mathbf{1 4}$

Page $10 \quad{ }^{1} \mathrm{H}-\mathrm{NMR}$ spectrum of $\mathbf{1}$

Page $11 \quad{ }^{1}$ H-NMR spectrum of 2

Page $12 \quad{ }^{1} \mathrm{H}-\mathrm{NMR}$ spectrum of $\mathbf{3}$

Page $13 \quad{ }^{1}$ H-NMR spectrum of 4 
Page $14 \quad{ }^{13} \mathrm{C}-\mathrm{NMR}$ spectrum of 4

Page $15 \quad{ }^{1}$ H-NMR spectrum of 5

Page $16 \quad{ }^{13} \mathrm{C}-\mathrm{NMR}$ spectrum of 5

Page $17 \quad{ }^{1} \mathrm{H}-\mathrm{NMR}$ spectrum of 7

Page $18 \quad{ }^{13} \mathrm{C}-\mathrm{NMR}$ spectrum of $\mathbf{7}$

Page $19 \quad{ }^{1}$ H-NMR spectrum of 8

Page $20 \quad{ }^{13} \mathrm{C}-\mathrm{NMR}$ spectrum of $\mathbf{8}$

Page $21 \quad{ }^{1} \mathrm{H}-\mathrm{NMR}$ spectrum of 9

Page $22 \quad{ }^{13} \mathrm{C}-\mathrm{NMR}$ spectrum of $\mathbf{9}$

Page $23 \quad{ }^{1} \mathrm{H}-\mathrm{NMR}$ spectrum of $\mathbf{1 0}$

Page $24 \quad{ }^{1} \mathrm{H}-\mathrm{NMR}$ spectrum of $\mathbf{1 1}$

Page $25 \quad{ }^{13} \mathrm{C}-\mathrm{NMR}$ spectrum of $\mathbf{1 1}$

Page $26 \quad{ }^{1} \mathrm{H}-\mathrm{NMR}$ spectrum of $\mathbf{1 2}$

Page $27 \quad{ }^{13} \mathrm{C}-\mathrm{NMR}$ spectrum of $\mathbf{1 2}$

Page $28 \quad{ }^{1}$ H-NMR spectrum of 14

Page $29 \quad{ }^{13} \mathrm{C}-\mathrm{NMR}$ spectrum of $\mathbf{1 4}$

Page $30 \quad \mathrm{X}$-ray structures of $\mathbf{1}$ and $\mathbf{5}$ 
Synthesis of 2<smiles>O=C(O)c1ccccc1</smiles><smiles>O=P(Cc1ccccc1)(c1ccccc1)c1ccccc1</smiles>

Following the typical procedure outlined for 1, chlorodiphenylphosphine $(1 \mathrm{~mL}, 5.46 \mathrm{mmol})$ was added to benzoic acid (222 mg, $1.82 \mathrm{mmol}$ ) and water (65 $\mu \mathrm{L}, 3.64 \mathrm{mmol})$. After microwave irradiation, work up and purification as outlined above, 2 was afforded as a white solid in a $79 \%$ yield (420 mg). Uncorrected melting point mp $189-193^{\circ} \mathrm{C}\left(\mathrm{Lit}^{1} 192-193^{\circ} \mathrm{C}\right),{ }^{1} \mathrm{H}$ NMR (400 MHz, $\left.\mathrm{CDCl}_{3}\right): \delta 3.66(\mathrm{~d}, 2 \mathrm{H}, J=13), 7.10-7.20(\mathrm{~m}, 5 \mathrm{H}), 7.40-7.54(\mathrm{~m}, 6 \mathrm{H}), 7.66-7.73(\mathrm{~m}, 4 \mathrm{H}) ;{ }^{13} \mathrm{C}$ NMR (75 MHz, $\left.\mathrm{CDCl}_{3}\right): \delta 133.1,131.9\left(\mathrm{~d} J_{\mathrm{PC}}=2.9\right), 131.2\left(\mathrm{~d} J_{\mathrm{PC}}=9.1\right), 130.2\left(\mathrm{~d} J_{\mathrm{PC}}=5.7\right), 128.6$, 128.5, 128.4, 126.8 (d $\left.J_{\mathrm{PC}}=2.9\right), 38.0\left(\mathrm{~d} J_{\mathrm{PC}}=66.0\right)$; IR $(\mathrm{KBr}) \vee 3058,1600,1492,1484,1453$, 1440, 1191, 1179, 1137, 1118, $1105 \mathrm{~cm}^{-1} ; \mathrm{m} / \mathrm{z}$ (EI+) 292.2 (14), 291.1 (33), 202.1 (13), 201.1 (100), 183.0 (4), 152.1 (4), 91.1 (41), 84.0 (18), 77.1 (26), 65.1 (14), 51.2 (19), 49.2 (27); HRMS calcd for $\mathrm{C}_{19} \mathrm{H}_{17} \mathrm{OP} 293.1090$, found 293.1091.

Synthesis of 3<smiles>Cc1ccccc1C(=O)O</smiles><smiles>Cc1ccccc1CP(=O)(c1ccccc1)c1ccccc1</smiles>

3

Following the typical procedure outlined for 1, chlorodiphenylphosphine $(1 \mathrm{~mL}, 5.46 \mathrm{mmol})$ was added to 2-toluic acid (248 mg, $1.82 \mathrm{mmol}$ ) and water (65 $\mu \mathrm{L}, 3.64 \mathrm{mmol})$. After microwave irradiation, work up and purification as outlined above, $\mathbf{3}$ was afforded as a white solid in a $71 \%$ yield (394 mg). Uncorrected melting point mp $123-125^{\circ} \mathrm{C}$, ( $\left.\mathrm{Lit}^{1} 122-123^{\circ} \mathrm{C}\right),{ }^{1} \mathrm{H} \mathrm{NMR}(400 \mathrm{MHz}$, $\left.\mathrm{CDCl}_{3}\right): \delta 2.14(\mathrm{~s}, 3 \mathrm{H}), 3.68(\mathrm{~d}, 2 \mathrm{H}, J=13), 7.07(\mathrm{~m}, 1 \mathrm{H}), 7.25-7.30(\mathrm{~m}, 2 \mathrm{H}), 7.39-7.85(\mathrm{~m}$, 20H); ${ }^{13} \mathrm{C}$ NMR (75 MHz, $\mathrm{CDCl}_{3}$ ): $\delta 134.2,133.8,133.6,132.7,132.0,131.3,131.2,131.1,130.3$, $128.6\left(\mathrm{~d} J_{\mathrm{PC}}=2.9\right), 128.4,37.1\left(\mathrm{~d} J_{\mathrm{PC}}=66.0\right)$; IR $(\mathrm{KBr}) \vee 1590,1493,1430,1405,1318,1238,1185$, 1100, $1170 \mathrm{~cm}^{-1}$; m/z (EI+) 342.2 (23), 217.1 (8), 202.1 (20), 201.1 (100), 183.0 (3), 141.0 (32), 
115.1 (28), 77.1 (18), 51.2 (6), 51.2 (19), 49.2 (27); HRMS calcd for $\mathrm{C}_{32} \mathrm{H}_{27}{ }^{79} \mathrm{BrO}_{2} \mathrm{P}_{2} 585.0742$, found 585.0740.

Synthesis of 4 .<smiles>O=C(O)c1ccc2ccccc2c1</smiles><smiles>O=P(Cc1ccc2ccccc2c1)(c1ccccc1)c1ccccc1</smiles>

4

Following the typical procedure outlined for 1, chlorodiphenylphosphine (1 mL, $5.46 \mathrm{mmol})$ was added to 2-naphthoic acid (313 mg, $1.82 \mathrm{mmol}$ ) and water (65 $\mu \mathrm{L}, 3.64 \mathrm{mmol})$. After microwave irradiation, work up and purification as outlined above, $\mathbf{4}$ was afforded as a white solid in a $68 \%$ yield (423 mg). Uncorrected melting point $\mathrm{mp}>250^{\circ} \mathrm{C},{ }^{1} \mathrm{H}$ NMR (400 MHz, $\left.\mathrm{CDCl}_{3}\right): \delta 3.84$ (d, $2 \mathrm{H}$, $J=13), 7.20-7.28(\mathrm{~m}, 2 \mathrm{H}), 7.40-7.77(\mathrm{~m}, 15 \mathrm{H}) ;{ }^{13} \mathrm{C} \mathrm{NMR}\left(75 \mathrm{MHz}, \mathrm{CDCl}_{3}\right): \delta 133.5\left(\mathrm{~d} J_{\mathrm{PC}}=\right.$ 3.1), 133.0, 132.4 (d $\left.J_{\mathrm{PC}}=2.3\right), 132.1\left(\mathrm{~d} J_{\mathrm{PC}}=3.1\right), 132.0,131.4\left(\mathrm{~d} J_{\mathrm{PC}}=9.1\right), 129.2\left(\mathrm{~d} J_{\mathrm{PC}}=6.8\right)$, $129.0\left(\mathrm{~d} J_{\mathrm{PC}}=7.6\right), 128.8\left(\mathrm{~d} J_{\mathrm{PC}}=12.2\right), 128.4\left(\mathrm{~d} J_{\mathrm{PC}}=4.6\right), 128.2\left(\mathrm{~d} J_{\mathrm{PC}}=2.3\right), 127.8\left(\mathrm{~d} J_{\mathrm{PC}}=8.3\right)$, 125.9, 126.0, 38.5 (d $\left.J_{\mathrm{PC}}=66.0\right)$; IR (KBr) $v$ 2935, 1510, 1438, $1185 \mathrm{~cm}^{-1} ; \mathrm{m} / \mathrm{z}(\mathrm{EI}+) 342.2$ (23), 217.1 (8), 202.1 (20), 201.1 (100), 183.0 (3), 141.0 (32), 115.1 (28), 77.1 (18), 51.2 (6), 51.2 (19), 49.2 (27); HRMS calcd for $\mathrm{C}_{23} \mathrm{H}_{19} \mathrm{OP} 343.1246$, found 343.1244.

Synthesis of 5.<smiles>O=C(O)c1ccccc1C(=O)O</smiles><smiles>O=P(Cc1ccccc1CP(=O)(c1ccccc1)c1ccccc1)(c1ccccc1)c1ccccc1</smiles>

Following the typical procedure outlined for 1 , chlorodiphenylphosphine $(1.67 \mathrm{~mL}, 9.10 \mathrm{mmol})$ was added to phthalic acid (302 mg, $1.82 \mathrm{mmol}$ ) and water (100 $\mu \mathrm{L}, 5.46 \mathrm{mmol}$ ). After microwave irradiation, work up and purification as outlined above, 5 was afforded as a white solid in a $62 \%$ yield (574 mg). Uncorrected melting point $\mathrm{mp}>250^{\circ} \mathrm{C},\left(\mathrm{Lit}^{2} 283^{\circ} \mathrm{C}\right){ }^{1} \mathrm{H}$ NMR $\left(400 \mathrm{MHz}, \mathrm{CDCl}_{3}\right): \delta$ $3.98(\mathrm{~d}, 4 \mathrm{H}, J=)$ ), $6.70-6.75(\mathrm{~m}, 2 \mathrm{H}), 6.84-6.88(\mathrm{~m}, 2 \mathrm{H}), 7.36-7.53(\mathrm{~m}, 12 \mathrm{H}), 7.64-7.73(\mathrm{~m}$, $8 \mathrm{H}) ;{ }^{13} \mathrm{C}$ NMR $\left(75 \mathrm{MHz}, \mathrm{CDCl}_{3}\right): \delta 133.3,132.0,131.8,131.7,131.6,131.3\left(\mathrm{~d}, J_{\mathrm{PC}}=9.1\right), 128.6(\mathrm{~d}$ 
$\left.J_{\mathrm{PC}}=11.9\right), 126.7,35.3\left(\mathrm{~d} J_{\mathrm{PC}}=66.0\right) ; \mathrm{IR}(\mathrm{KBr}) \vee 2949,1599,1485,1435,1411,1180,1151,1122$, $1103 \mathrm{~cm}^{-1} ; \mathrm{m} / \mathrm{z}$ (EI+) 506.2 (17), 382.2 (7), 321.2 (11), 305.1 (10), 201.1 (82), 181.1 (22), 166.1 (11), 104.1 (13), 84.0 (26), 77.1 (42), 49.2 (37), 44.2 (100); HRMS calcd for $\mathrm{C}_{32} \mathrm{H}_{28} \mathrm{O}_{2} \mathrm{P}_{2}$ 507.1637, found 507.1636

Synthesis of $\mathbf{7}$.<smiles>O=C(O)c1ccc(Br)c(C(=O)O)c1</smiles><smiles>O=P(Cc1ccccc1)(Cc1cccc(Br)c1)c1ccccc1</smiles>

Following the typical procedure outlined for 1, chlorodiphenylphosphine (7.48 mL, $40.81 \mathrm{mmol})$ was added to 4-bromoisophthalic acid (2g, $8.61 \mathrm{mmol})$ and water (440 $\mu \mathrm{L}, 24.49 \mathrm{mmol})$. After microwave irradiation, work up and purification as outlined above, 7 was afforded as a white solid in a 54\% yield (2.58g). Uncorrected melting point $\mathrm{mp}>250^{\circ} \mathrm{C},{ }^{1} \mathrm{H}$ NMR $\left(400 \mathrm{MHz}, \mathrm{CDCl}_{3}\right): \delta 3.62$ (d, $2 \mathrm{H}, J=13), 3.77$ (d, 2H, $J=13), 7.07(\mathrm{~m}, 1 \mathrm{H}), 7.25-7.30(\mathrm{~m}, 2 \mathrm{H}), 7.39-7.85(\mathrm{~m}, 20 \mathrm{H}) ;{ }^{13} \mathrm{C}$ NMR (75 MHz, $\mathrm{CDCl}_{3}$ ): $\delta$ 134.2, 133.9, 133.6, 132.7, 132.0, 131.3, 131.2, 131.1, 130.3, 128.6 (d $\left.J_{\mathrm{PC}}=2.9\right), 128.4,37.1\left(\mathrm{~d} J_{\mathrm{PC}}=66.0\right) ; \mathrm{IR}(\mathrm{KBr}) \vee 2946,1522,1493,1436,1160 \mathrm{~cm}^{-1} ; \mathrm{m} / \mathrm{z}(\mathrm{EI}+)$ 342.2 (23), 217.1 (8), 202.1 (20), 201.1 (100), 183.0 (3), 141.0 (32),15.1 (28), 77.1 (18), 51.2 (6), 51.2 (19), 49.2 (27); HRMS calcd for $\mathrm{C}_{32} \mathrm{H}_{27}{ }^{79} \mathrm{BrO}_{2} \mathrm{P}_{2}$ 585.0742, found 585.0740 Microwave procedure for the synthesis of $\mathbf{8}$<smiles></smiles>

7

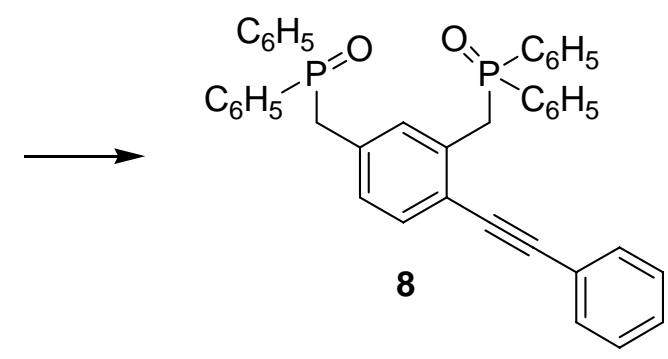

A mixture of 7 (300 mg, 0.5mmol), phenylacetylene (0.07mL, $0.61 \mathrm{mmol}), \mathrm{PdCl}_{2}\left(\mathrm{PPh}_{3}\right)_{2}(17.9 \mathrm{mg}$, $0.025 \mathrm{mmol}, 5 \mathrm{~mol} \%)$, CuI (4.7 mg, $0.025 \mathrm{mmol}, 5 \mathrm{~mol} \%)$, $\left({ }^{\mathrm{i}} \mathrm{Pr}\right)_{2} \mathrm{NH}(0.2 \mathrm{~mL}),{ }^{\mathrm{t}} \mathrm{Bu}_{3} \mathrm{P}(10.3 \mathrm{mg}, 1 \mathrm{M}$ solution in toluene, $10 \mathrm{~mol} \%)$ in $\mathrm{DMF}(2 \mathrm{~mL})$ was stirred under argon in a sealed (aluminum crimp cap) Smith reaction tube for $10 \mathrm{~min}$, after which the reaction was heated in a Personal Chemistry 
microwave synthesizer at $120^{\circ} \mathrm{C}$ for $30 \mathrm{~min}$. The mixture was cooled to room temperature and diluted with water $(10 \mathrm{~mL})$. The reaction products were extracted with ethyl acetate $(3 \times 5 \mathrm{~mL})$. The combined ethyl acetate extracts were washed successively with water ( 2 x $5 \mathrm{~mL}$ ), dilute $\mathrm{HCl}$ ( $5 \mathrm{~mL}$ ), water $(5 \mathrm{~mL})$ and brine $(5 \mathrm{~mL})$. The organic phase was dried over anhydrous $\mathrm{MgSO}_{4}$, filtered and solvent removed under reduced pressure. Flash column chromatography using dichloromethanemethanol $(3 \% \mathrm{MeOH})$ solvent system afforded the product as pale brown oil (278 mg, 90\%). $v_{\max }$ FT-IR (film / cm ${ }^{-1}$ ) 3429, 3055, 2907, 1595, 1499, 1437; $\delta_{\mathrm{H}}\left(400 \mathrm{MHz}, \mathrm{CDCl}_{3}\right) 3.64$ (2H, d, $\left.J_{P H} 14\right)$, 3.84 (2H, d, $\left.J_{P H} 14\right), 7.13$ (1H, d, $J$ 8.1), 7.20 (1H, d, $J$ 8.1), 7.31 - 7.70 (26H, m, ArH); $\delta_{\mathrm{C}}(100$ $\left.\mathrm{MHz}, \mathrm{CDCl}_{3}\right) 36.2$ (d, $J_{P C} 65$ ), 38.2 (d, $J_{P C} 65$ ), 88.0, 93.7, 123.2, 128.5, 128.7, 128.8, 128.9, 131.2, 131.3, 131.4, 131.5 (d, $\left.J_{P C} 16\right), 131.9$ (d, $\left.J_{P C} 16\right), 132.0,132.1,132.2,132.3,133.4,132.6,132.7$, 132.8; $\mathrm{m} / \mathrm{z}(\mathrm{CI}) 607.0[\mathrm{M}+\mathrm{H}]^{+}$(100), $707.3\left[\mathrm{M}+\mathrm{Pr}_{2} \mathrm{NH}\right]^{+}(22), 810.5\left[\mathrm{M}+\left(\mathrm{iPr}_{2} \mathrm{NH}\right)_{2}\right]^{+}(20) ; \mathrm{HRMS}$ calcd for $\mathrm{C}_{40} \mathrm{H}_{32} \mathrm{O}_{2} \mathrm{P}_{2}$ 607.1950, found 607.1950

Conventional heating procedure for the Sonagashira synthesis of $\mathbf{9}$

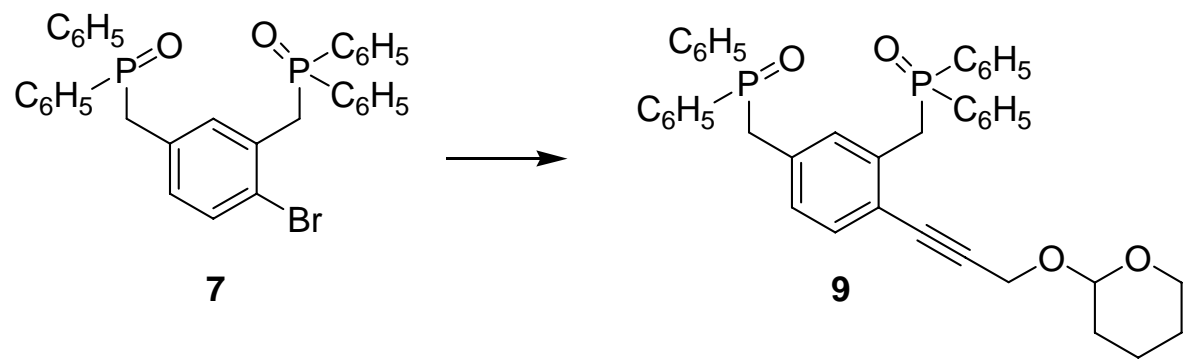

A mixture of 7 (200 mg, $0.34 \mathrm{mmol}$ ), $O$-THP protected propargyl alcohol (56 mg, $0.41 \mathrm{mmol}$ ), $\mathrm{PdCl}_{2}\left(\mathrm{PPh}_{3}\right)_{2}$ (12 mg, $0.017 \mathrm{mmol}, 5 \mathrm{~mol} \%$ ), CuI (3.3 mg, $\left.0.017 \mathrm{mmol}, 5 \mathrm{~mol} \%\right),{ }^{\mathrm{t}} \mathrm{Bu}_{3} \mathrm{P}$ (6.8 mg, $1 \mathrm{M}$ solution in toluene, $10 \mathrm{~mol} \%)$ was added to freshly distilled and degassed $\mathrm{Et}_{3} \mathrm{~N}(2 \mathrm{~mL})$ and stirred under argon. The reaction was heated at $60^{\circ} \mathrm{C}$ for 12 hours, cooled to room temperature and the solvent evaporated under reduced pressure and water added $(10 \mathrm{~mL})$. The aqueous phase was extracted with ethyl acetate ( 3 x $5 \mathrm{~mL}$ ). The combined ethyl acetate extracts were washed successively with water ( $2 \times 5 \mathrm{~mL})$ and brine $(5 \mathrm{~mL})$. The organic phase was dried over anhydrous $\mathrm{MgSO}_{4}$, filtered and solvent removed under reduced pressure. Flash column chromatography using dichloromethane-methanol ( $3 \% \mathrm{MeOH})$ as the eluent solvent system afforded $\mathbf{9}$ as a pale brown oil (195 mg, 88 \%). v $v_{\max }$ FT-IR (film / cm ${ }^{-1}$ ) 3412, 3044, 2948, 2894, 1476, 1437, 1193; $\delta_{\mathrm{H}}(300 \mathrm{MHz}$, $\left.\mathrm{CDCl}_{3}\right) 1.27$ - $1.73(6 \mathrm{H}, \mathrm{m}), 3.50$ (2H, d, $\left.J_{P H} 13.5\right), 3.57-3.72(4 \mathrm{H}, \mathrm{m}), 4.23(1 \mathrm{H}, \mathrm{d}, J 15.8), 4.34$ (1H, d, $J$ 15.8), 4.72 (1H, s), 6.91 (1H, d, $J$ 2.8), 7.01 (1H, dd, $J 8.2$ and 2.8), 7.13 (1H, d, $J$ 8.2), 6.28 - 7.62 (20H, m, ArH); $\delta_{\mathrm{C}}\left(75 \mathrm{MHz}, \mathrm{CDCl}_{3}\right)$ 18.8, 25.1, 28.8, 30.1, 36.6 (d, JPC 65.3), 37.5 (d, $J_{P C}$ 65.3), 54.6, 61.9, 84.1, 89.3, 96.8, 121.5, 123.7, 128.2, 128.3, 128.5, 128.7, 130.2, 130.9 (d, $J_{P C}$ 
9.7), 131.2 (d, $J_{P C}$ 9), 131.4, 131.8, 132.2, 132.6, 133.7; m/z $\left(\mathrm{ES}^{+}\right) 644.7\left(\mathrm{M}^{+}\right)$; HRMS calcd for $\mathrm{C}_{40} \mathrm{H}_{38} \mathrm{O}_{4} \mathrm{P}_{2}$ 645.2318, found 645.2317

Synthesis of $\mathbf{1 0}$

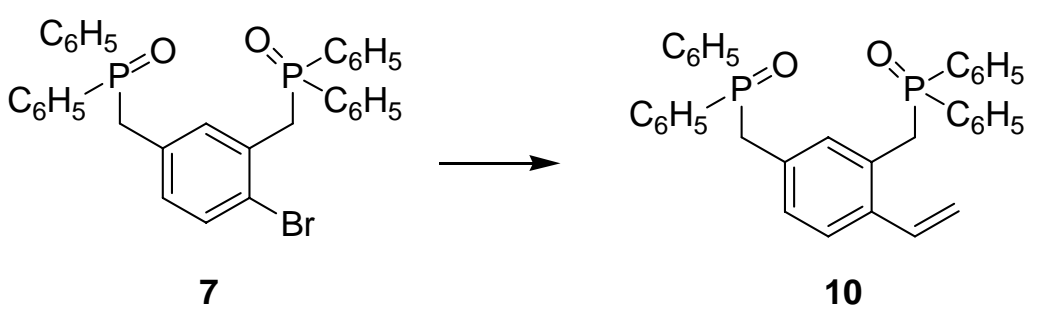

To a solution of 7 (2g, $3.42 \mathrm{mmol})$, vinyl tri-n-butyltin $(1.1 \mathrm{~mL}, 3.76 \mathrm{mmol})$ in dry toluene $(35 \mathrm{~mL})$ under an inert atmosphere was added tris(dibenzylideneacetone)palladium(0) (156 mg, $0.17 \mathrm{mmol}$ ) and triphenylphosphine $(179 \mathrm{mg}, 0.68 \mathrm{mmol})$. The reaction was heated at $120^{\circ} \mathrm{C}$ for 24 hours, followed by removal of the solvent in vacuo. The oily residue was redissolved in dichloromethane $(50 \mathrm{~mL})$, filtered through a Celite pad and then washed with dichloromethane (50 mL). The organic solution was washed with $10 \%$ aqueous ammonia $(3 \times 50 \mathrm{~mL})$, water $(3 \times 50 \mathrm{~mL})$, dried over magnesium sulphate, filtered and concentrated in vacuo. The product was purified by flash column chromatography on silica gel (dichloromethane / methanol 97 : 3) affording 10 as a white solid (920 mg, 51\%). Mp decomposed $>80^{\circ} \mathrm{C}$, $v_{\max }$ FT-IR (film / cm $\left.{ }^{-1}\right) 3437 ; \delta_{\mathrm{H}}\left(400 \mathrm{MHz}, \mathrm{CDCl}_{3}\right) 3.48(2 \mathrm{H}$, d, $\left.J_{P H} 14\right), 3.55$ (2H, d, $\left.J_{P H} 14\right), 4.99$ (1H, d, $J$ 11), 5.26 (1H, d, $J$ 17), 6.63 (1H, dd, $J 11$ and 17), 6.87 - 7.67 (23H, m, ArH); $\delta_{C}\left(100 \mathrm{MHz}, \mathrm{CDCl}_{3}\right) 35$ (d, $\left.J_{P C} 65\right), 37.7$ (d, $\left.J_{P C} 65\right), 116.5,126.6$, 128.6, 128.7, 128.8, 128.9, 129.1, 131.3 (d, $J_{P C}$ 9), 131.3, 131.4, 131.5, 131.7, 132.0, 132.7, 132.9, 133.3, 134.2; m/z (CI/NH $) 532.2\left(\mathrm{M}^{+}, 6 \%\right), 408$, (4), 331 (18), 202 (15), 201 (100), 183 (18), 173 (11), 152 (8), 128 (20), 115 (14), 78 (10), 77 (39), 51 (24); HRMS calcd for $\mathrm{C}_{40} \mathrm{H}_{34} \mathrm{O}_{2} \mathrm{P}_{2} 609.2107$, found 609.2115.

Synthesis of $\mathbf{1 1}^{3}$<smiles>O=P(O)(c1ccccc1)c1ccc(P(=O)(Cc2ccccc2)c2ccccc2)cc1</smiles>

7

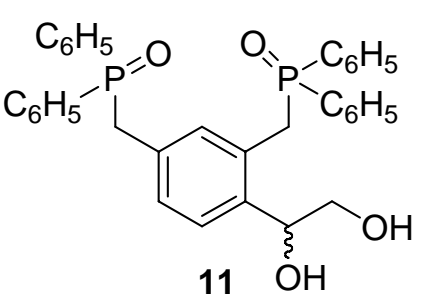

$11 \mathrm{OH}$

To a solution of 10 (550 mg, $1.03 \mathrm{mmol})$, potassium hexacyanoferrate(III) (1.02 g, $3.10 \mathrm{mmol}$ ), potassium carbonate (430 mg, $3.10 \mathrm{mmol}$ ), and quinuclidine (34 mg, $0.31 \mathrm{mmol}$ ) in a mixture of water / tert-butanol ( $1: 1,7 \mathrm{~mL}$ each) was added osmium(III) chloride (5 mg, $0.017 \mathrm{mmol})$. The 
resulting mixture was stirred vigorously at room temperature until TLC indicated consumption of the starting material. Sodium sulfite $(2.08 \mathrm{~g}, 16.54 \mathrm{mmol})$ was added and the stirring maintained for a further $1 \mathrm{~h}$ before the addition of dichloromethane $(10 \mathrm{~mL})$. The aqueous phase was further extracted with dichloromethane $\left(3 \times 10 \mathrm{~mL}\right.$ ). The combined organic phases were dried over $\mathrm{MgSO}_{4}$ and evaporated in vacuo. The residue was purified using flash column chromatography on silica gel (dichloromethane / methanol, 95 : 5) affording the product as a white solid (480 mg, 52\%). mp $123^{\circ} \mathrm{C}$. FT-IR (thin layer): 3409, 2227, 1640, 1435, 1176, $1120 \mathrm{~cm}^{-1} ; \delta_{\mathrm{H}}\left(300 \mathrm{MHz}, \mathrm{CDCl}_{3}\right) 2.95$ $(\mathrm{OH}), 3.20\left(1 \mathrm{H}, \mathrm{t}, J_{P H} 14\right.$ and $\left.J_{H H} 14\right), 3.34$ (1H, dd, $J_{P H} 15$ and $\left.\mathrm{J}_{\mathrm{HH}} 13\right)$, 3.55-3.69 (2H, m), 3.793.93 (2H, m), 4.93 (1H, dd, $J 10$ and 8), $5.25(\mathrm{OH}), 6.46$ (1H,d, $J$ 3), 6.73 (1H, dd, $J 11$ and 3), 7.19 (1H, d, $J$ 11), 7.35 - 7.63 (18H, m, ArH), 7.73 (t, 2H, $J$ 11) ; $\delta_{\mathrm{C}}\left(75 \mathrm{MHz}, \mathrm{CDCl}_{3}\right) 34.4$ (d, JPC 65), 36.3 (d, $\left.J_{P C} 65\right)$, 65.2, 69.3,126.46 (d, $J_{P C}$ 2), 126.50 (d, $J_{P C}$ 3), 127.66, 127.73, 127.8, 127.9, 128.0, 128.1 (d, $\left.J_{P C} 3\right), 128.1,128.2$ (d, $\left.J_{P C} 3\right), 128.4$ (d, $\left.J_{P C} 3\right), 128.5$ (d, $\left.J_{P C} 3\right), 129.2,129.5$ (d, $\left.J_{P C} 3\right)$, 129.6 (d, $J_{P C}$ 3), 130.18 (d, $J_{P C} 3$ ), 130.26 (d, $J_{P C} 3$ ), 130.34 (d, $J_{P C} 3$ ), 130.5, 130.6, 131.0, 131.1 (d, $J_{P C}$ 3), 131.3 (d, $J_{P C}$ 3), 131.5 (d, $J_{P C}$ 3), 131.9, 132.3, 132.7 (d, $J_{P C}$ 5), 132.8 (d, $J_{P C}$ 5), 139.36 (d, $J_{P C}$ 4), 139.43 (d, $\left.J_{P C} 4\right) ; m / z(L C / M S) 566.8\left(\mathrm{M}^{+}\right)$, 548.8, 304, 279.9, 142.95. HRMS calcd for $\mathrm{C}_{34} \mathrm{H}_{32} \mathrm{O}_{4} \mathrm{P}_{2}$ 567.1849, found 567.1848.

Synthesis of 12
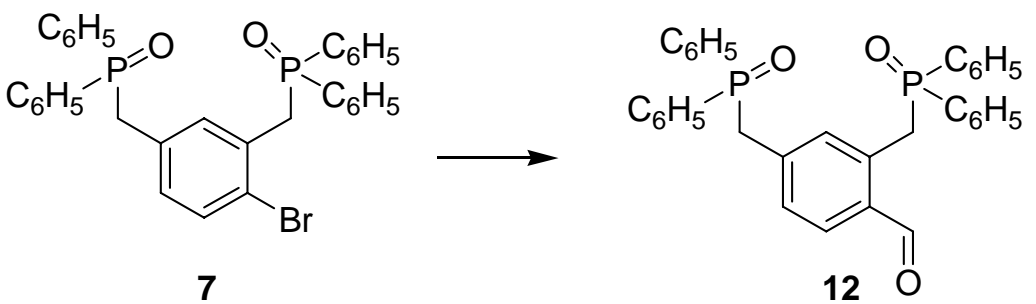

A solution of sodium periodate (340 mg, $1.59 \mathrm{mmol}$ ) in water (1.5 mL) was added to silica gel (3 g) and the solid stirred for $1 \mathrm{~h}$. The resulting silica was added to a solution of 11 (450 mg, $0.79 \mathrm{mmol}$ ) in DCM (15 mL) and the suspension stirred vigorously at room temperature for $2 \mathrm{~h}$, after which time TLC indicated complete consumption of 11. Filtering the reaction and evaporation of the solvent in vacuo afforded 12 as a clear oil (325 mg, 76\%). $v_{\max }$ FT-IR (film / cm ${ }^{-1}$ ) 1694; $\delta_{\mathrm{H}}(400 \mathrm{MHz}$, $\left.\mathrm{CDCl}_{3}\right) 3.72$ (2H, d, $\left.J_{P H} 14\right), 4.23$ (2H, d, $\left.J_{P H} 15\right), 7.27$ - 7.74 (23H, m, ArH), 9.65 (1H, s, CHO); $\delta_{\mathrm{C}}$ $\left(75 \mathrm{MHz}, \mathrm{CDCl}_{3}\right) 33.5$ (d, $J_{P C}$ 63), 37.1 (d, $\left.J_{P C} 66\right), 128.3,128.4,128.6$ (d, $\left.J_{P C} 3\right), 128.7,128.8$, 128.9, 130.2, 131.0, 131.1 (d, $J_{P C}$ 9), 131.2, 131.3 (d, $J_{P C}$ 6), 131.4, 132.0, 132.1, 132.5, 132.7, 134.8, 192.8; m/z (CI/NH 3 ) 535.1 (M+, 30\%), 522, (20), 507 (10), 220 (70), 203 (100), 187 (20), 96 (50); HRMS: found $\left(\mathrm{ES}^{+}\right)[\mathrm{M}+\mathrm{H}]^{+}$535.1587. $\mathrm{C}_{33} \mathrm{H}_{28} \mathrm{O}_{3} \mathrm{P}_{2}$ requires $[\mathrm{M}+\mathrm{H}]^{+}$535.1586. 
Synthesis of 14<smiles>CP(=O)(Cc1ccccc1)Cc1cc(CP(C)(=O)c2ccccc2)ccc1Br</smiles>

7

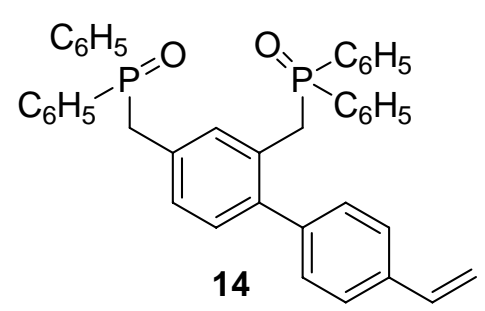

14

To a degassed solution of 7 (200 mg, $0.27 \mathrm{mmol}$ ) and para-vinylphenylboronic acid (49 mg, 0.33 mmol) in a mixture of toluene : ethanol : water ( $10: 2: 1 \mathrm{~mL}$ respectively) was added potassium carbonate (491 mg, $3.55 \mathrm{mmol}$ ) and tetrakis(triphenylphosphine)palladium(0) (32 mg, $0.27 \mathrm{mmol}$ ). The resulting mixture was heated at $85^{\circ} \mathrm{C}$ under an atmosphere of argon for 16 hours. The reaction was cooled to ambient temperature, partioned between dichloromethane / water and extracted with aliquots (3 x $10 \mathrm{~mL}$ ) of dichloromethane. The combined organic layers were dried $\left(\mathrm{MgSO}_{4}\right)$, filtered and the solvent removed in vacuo. Flash column chromatography on silica gel, using 5\% methanol in dichloromethane afforded the product as clear oil (94 mg, 56\% yield). $v_{\max }$ FT-IR (film / cm ${ }^{-1}$ ) 3426, 3057, 2221, 1629, 1489, 1437, 1119; $\delta_{\mathrm{H}}\left(400 \mathrm{MHz}, \mathrm{CDCl}_{3}\right) 3.52$ (2H, d, $\left.J_{P H} 14\right), 3.60(2 \mathrm{H}, \mathrm{d}$, $\left.J_{P H} 14\right), 5.20$ (1H, d, $J$ 11), 5.70 (1H, d, $J$ 17), 6.63 (1H, dd, $J 11$ and 17), $6.73-7.67$ (27H, m, $\mathrm{ArH}) ; \delta_{\mathrm{C}}\left(100 \mathrm{MHz}, \mathrm{CDCl}_{3}\right) 32$ (d, $\left.J_{P C} 66\right), 35.6$ (d, $\left.J_{P C} 66\right), 112.2,124.2,128.3,128.4,128.5$, 128.7, 129.6, 130.4, 131.0, 131.1, 131.2, 131.3, 131.6, 131.7, 132.9, 133.1; m/z (LC/MS) 609.1, 582.75, 557, 540, 506, 454.7, 422.7, 310.7 
mus 5009

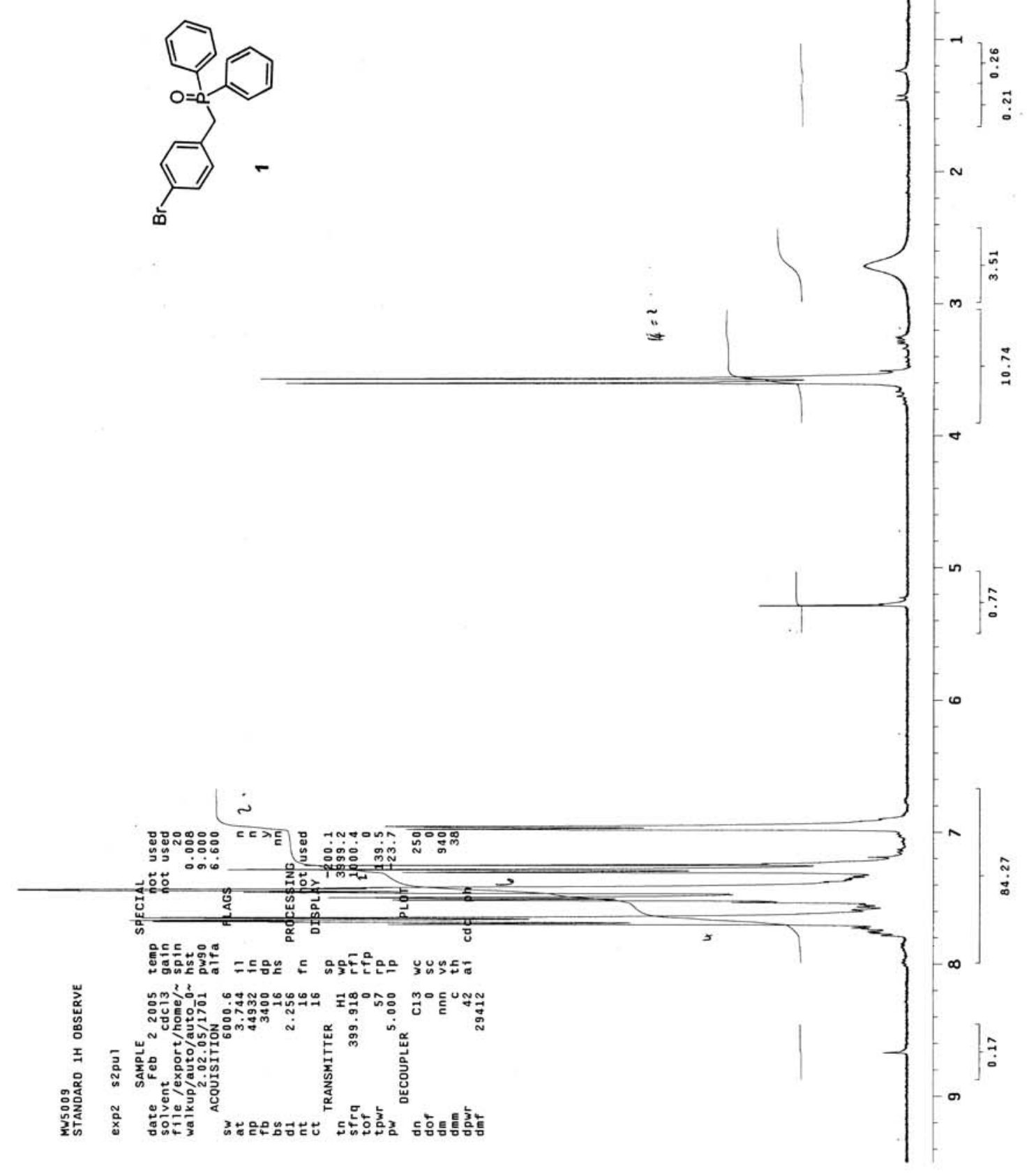




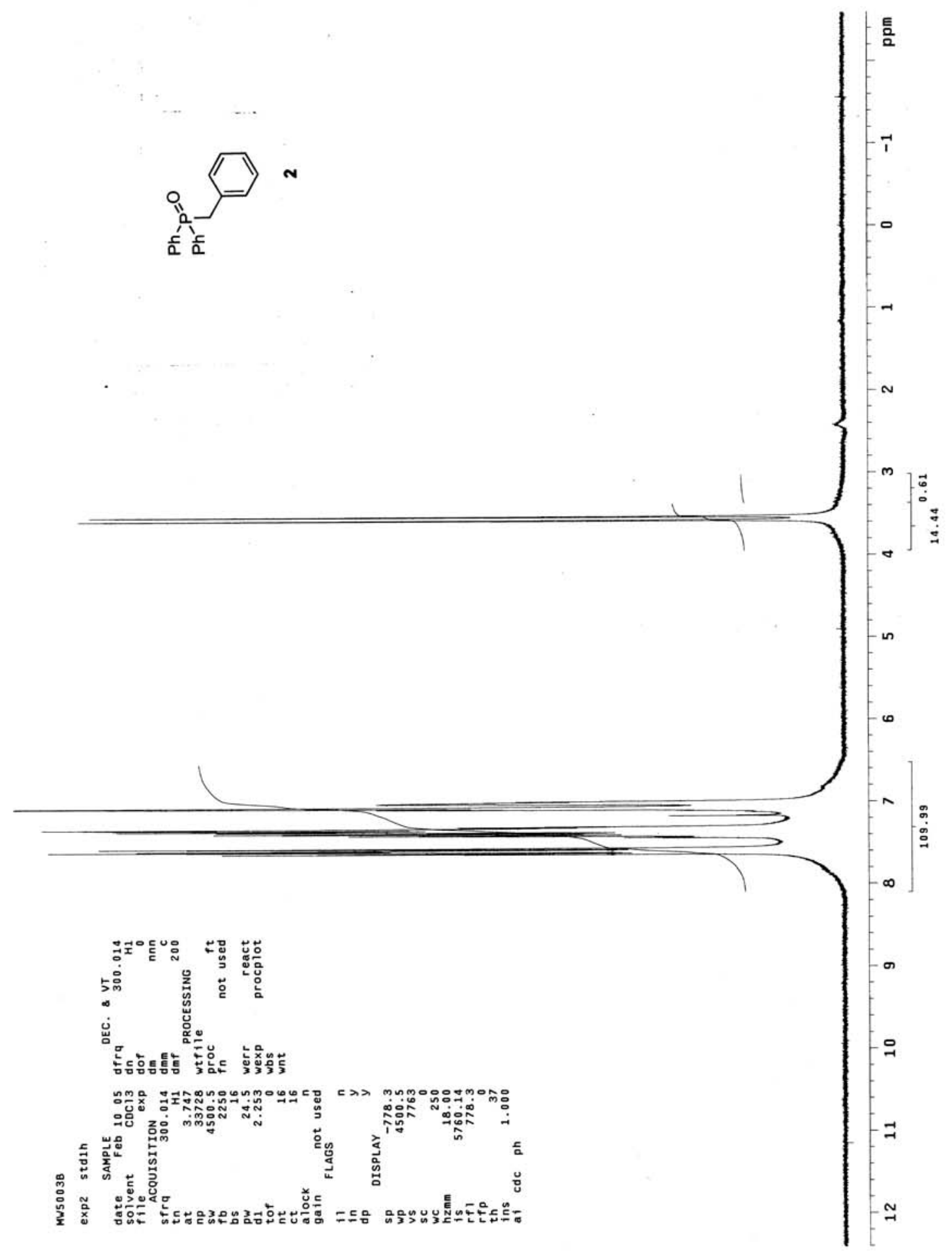




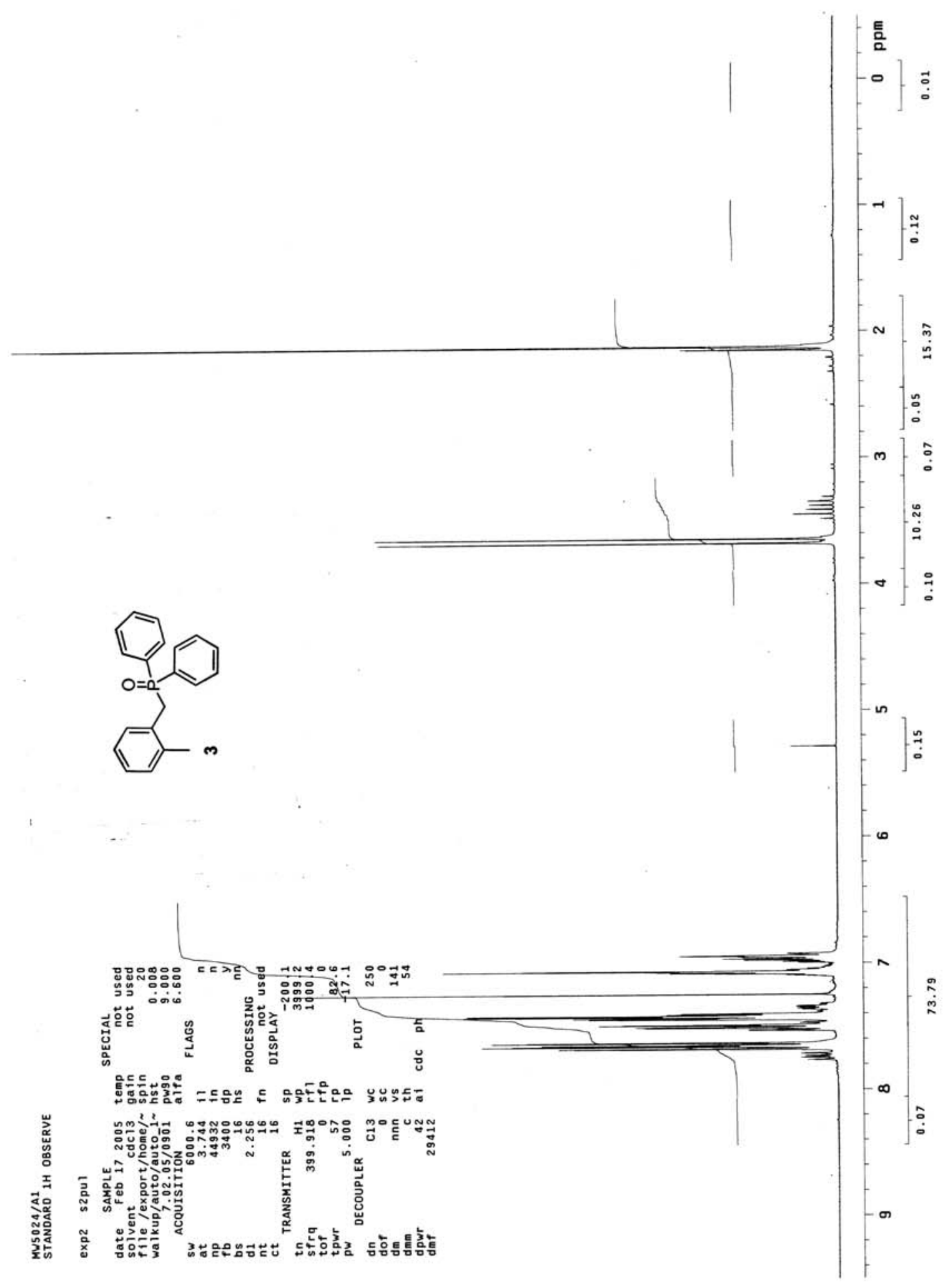




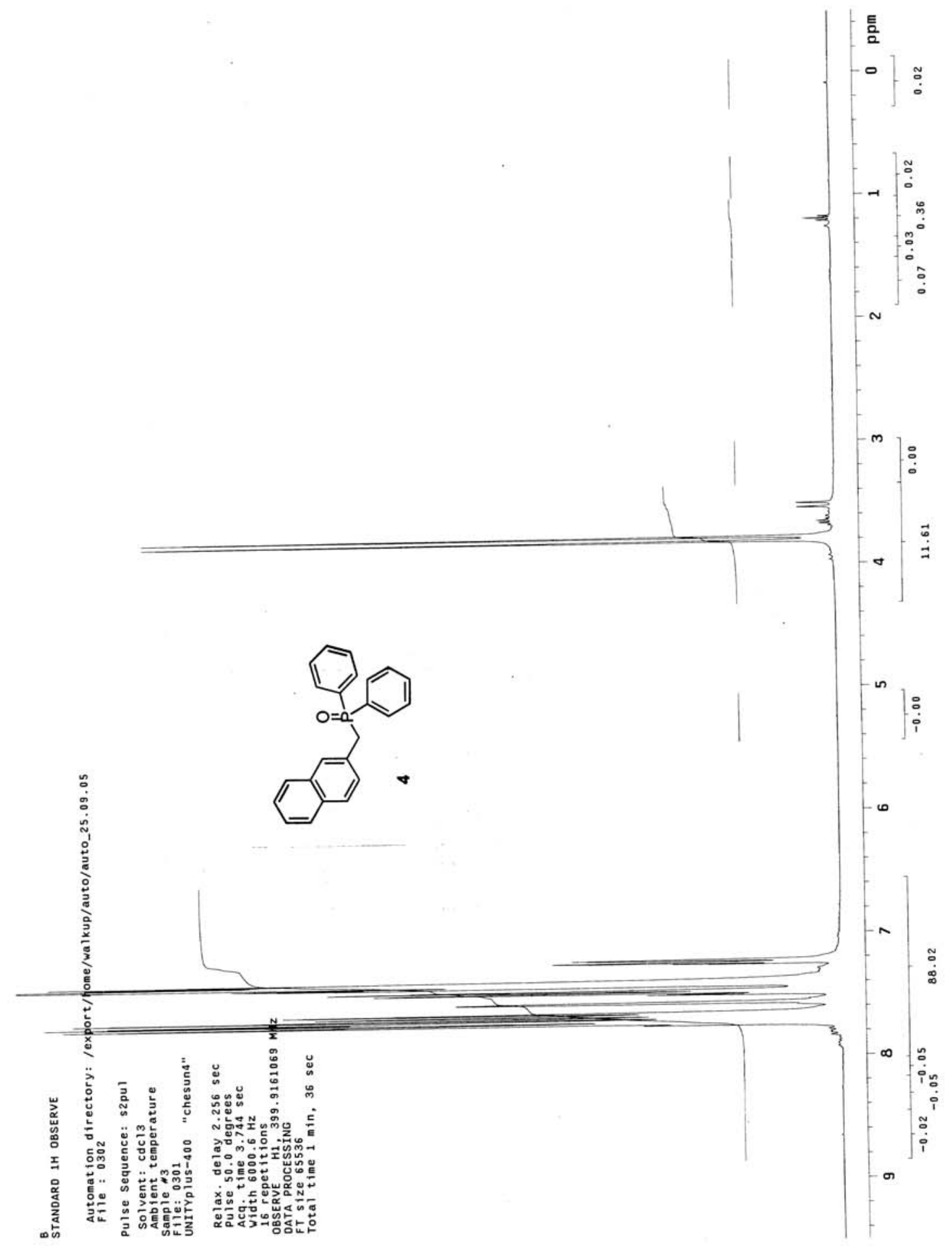




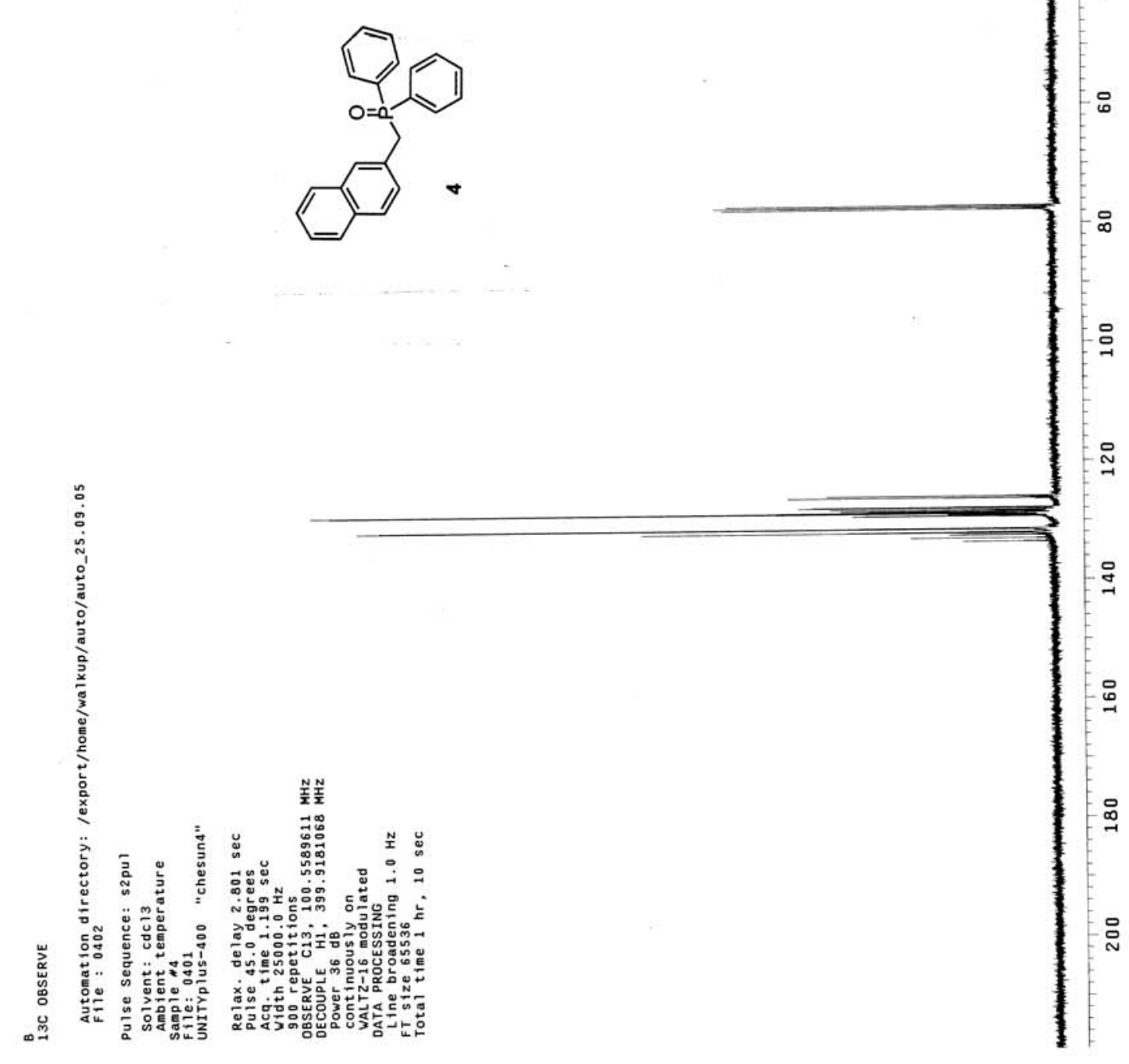


mus5007

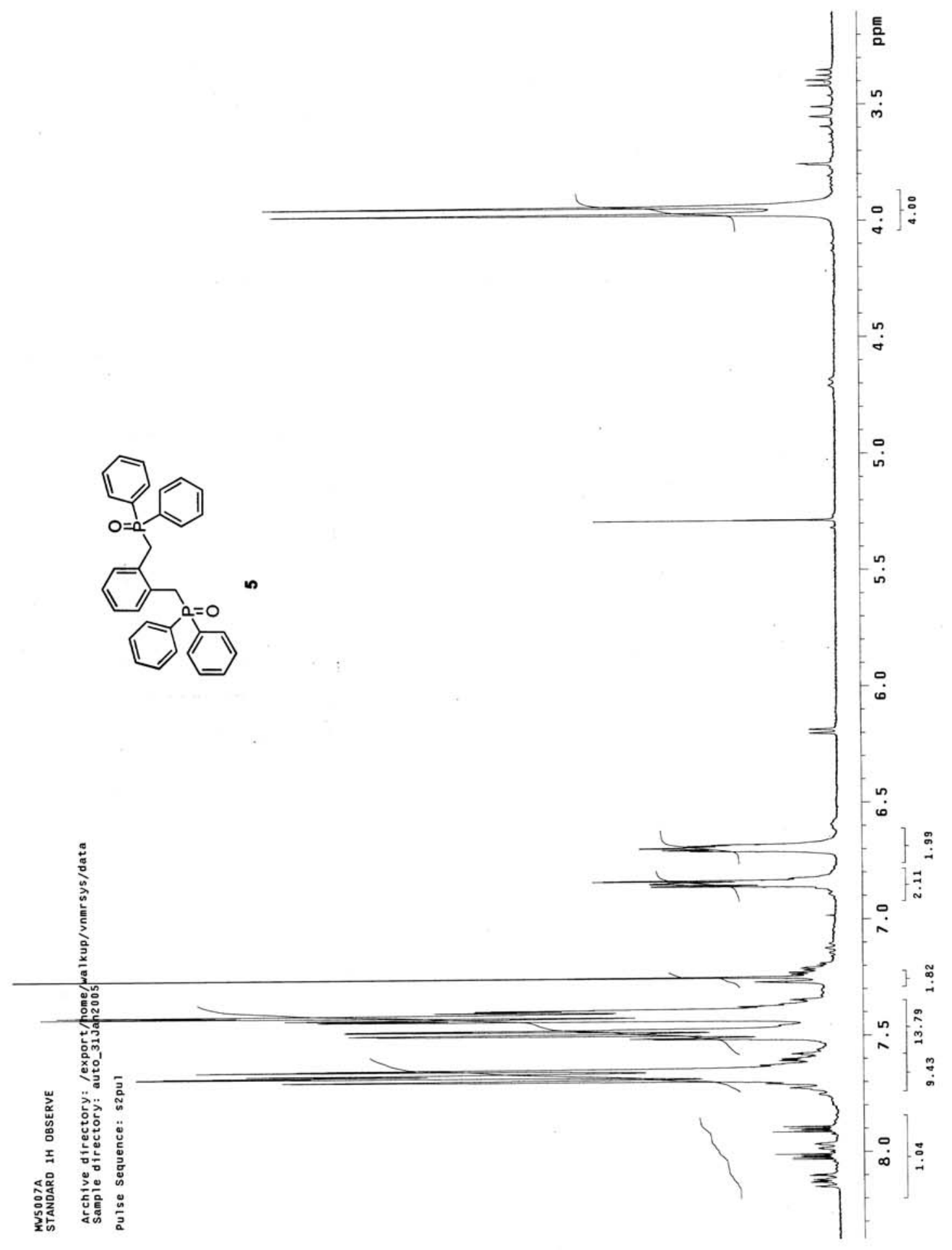


Mw 5007

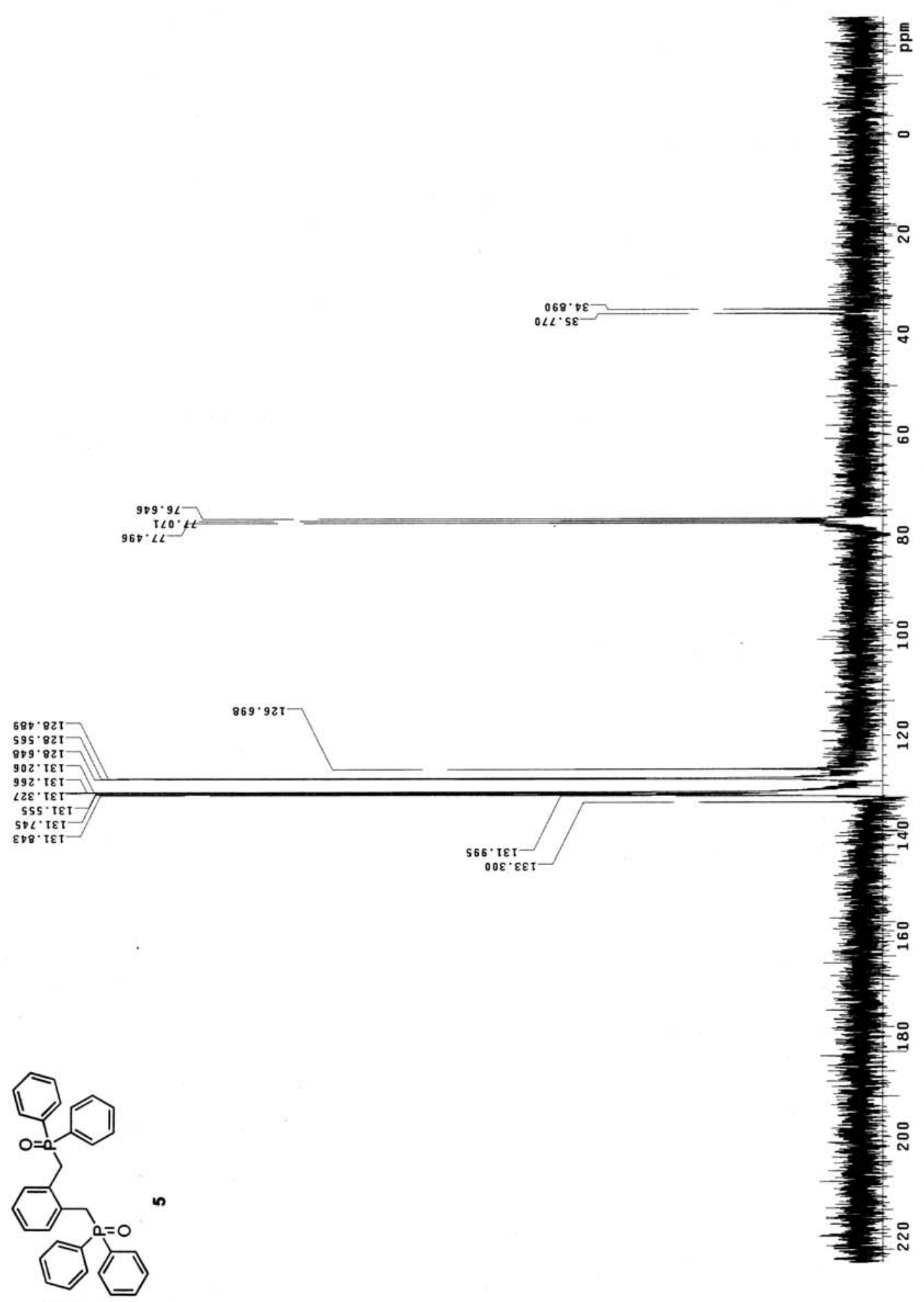




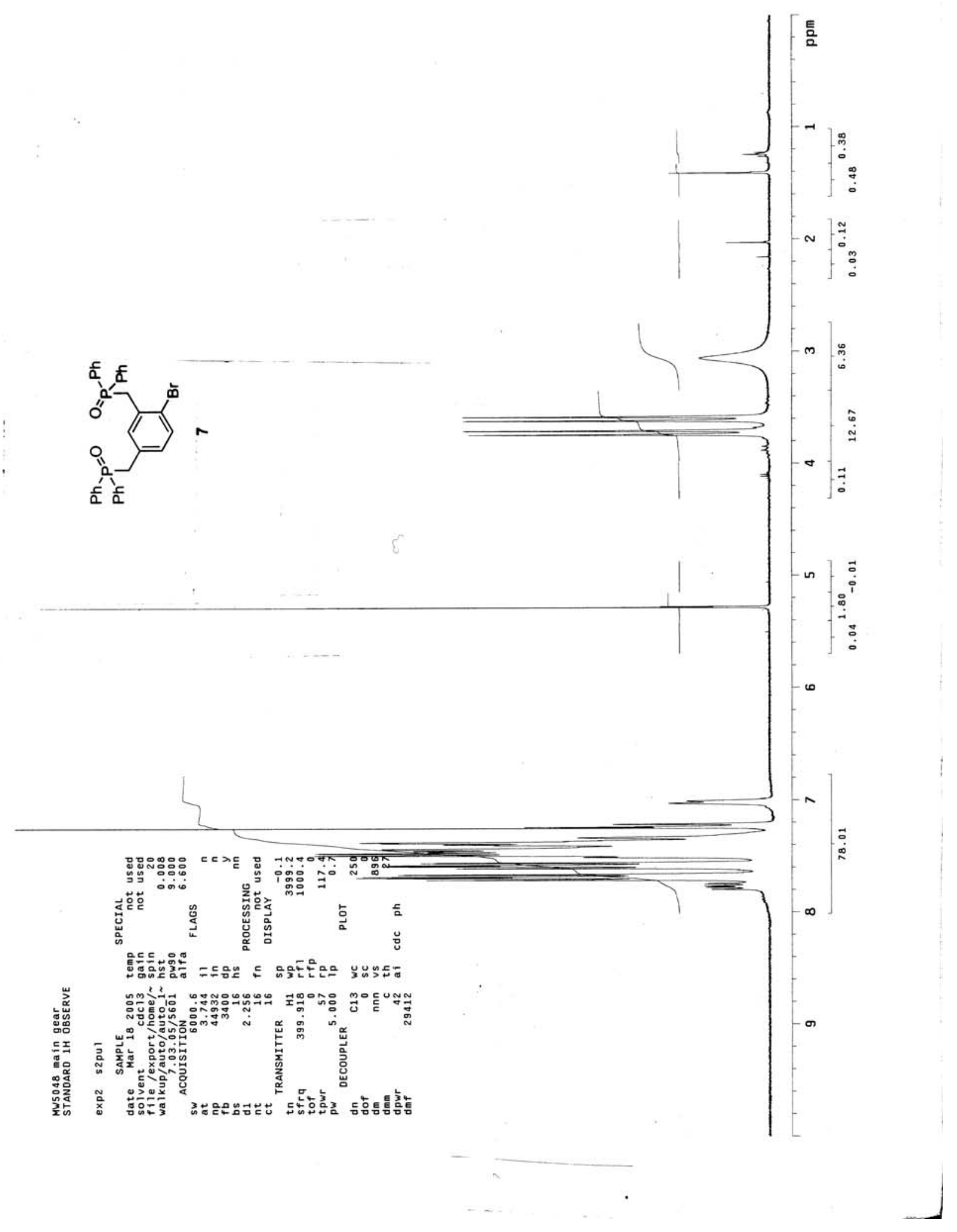




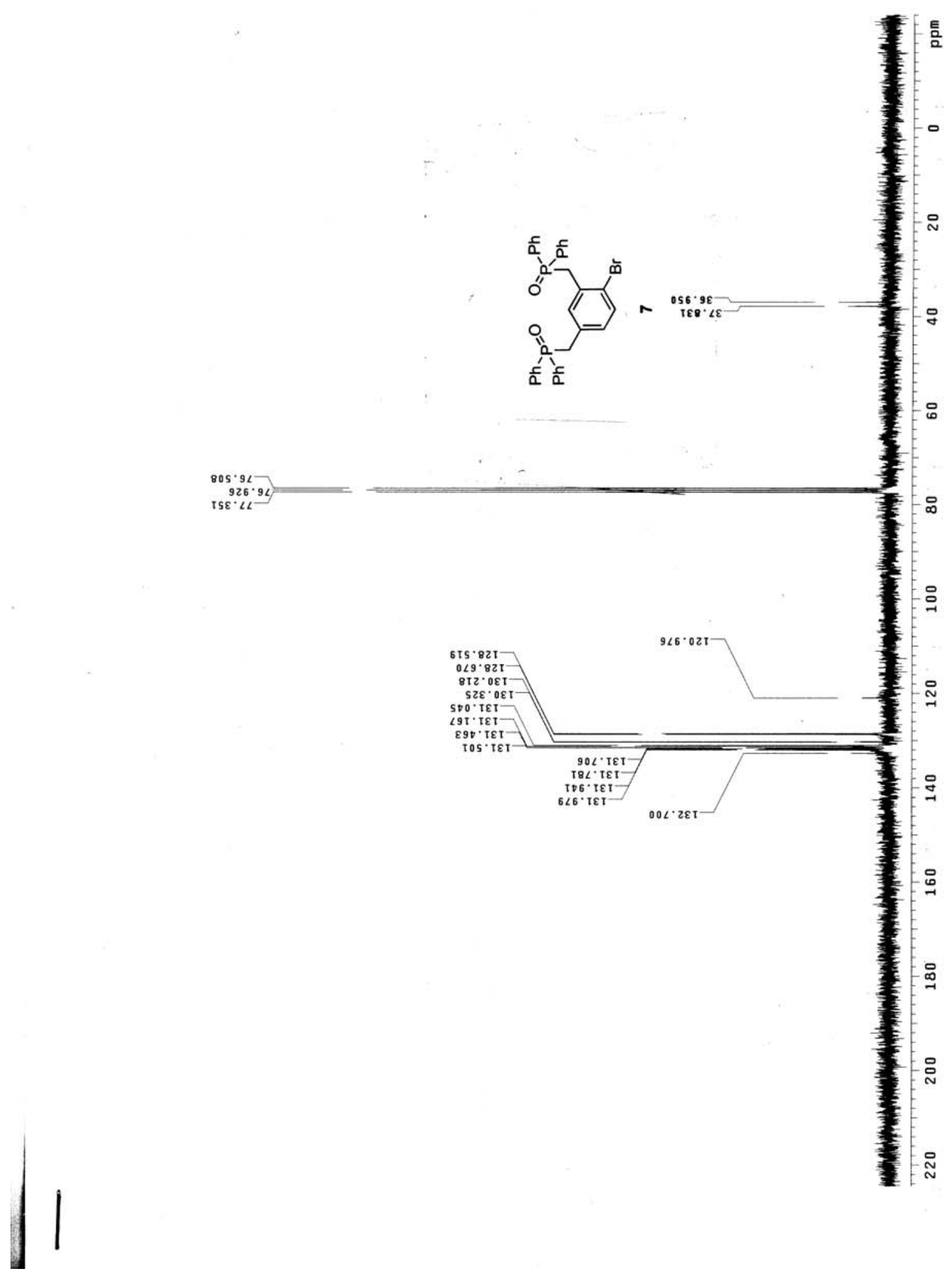




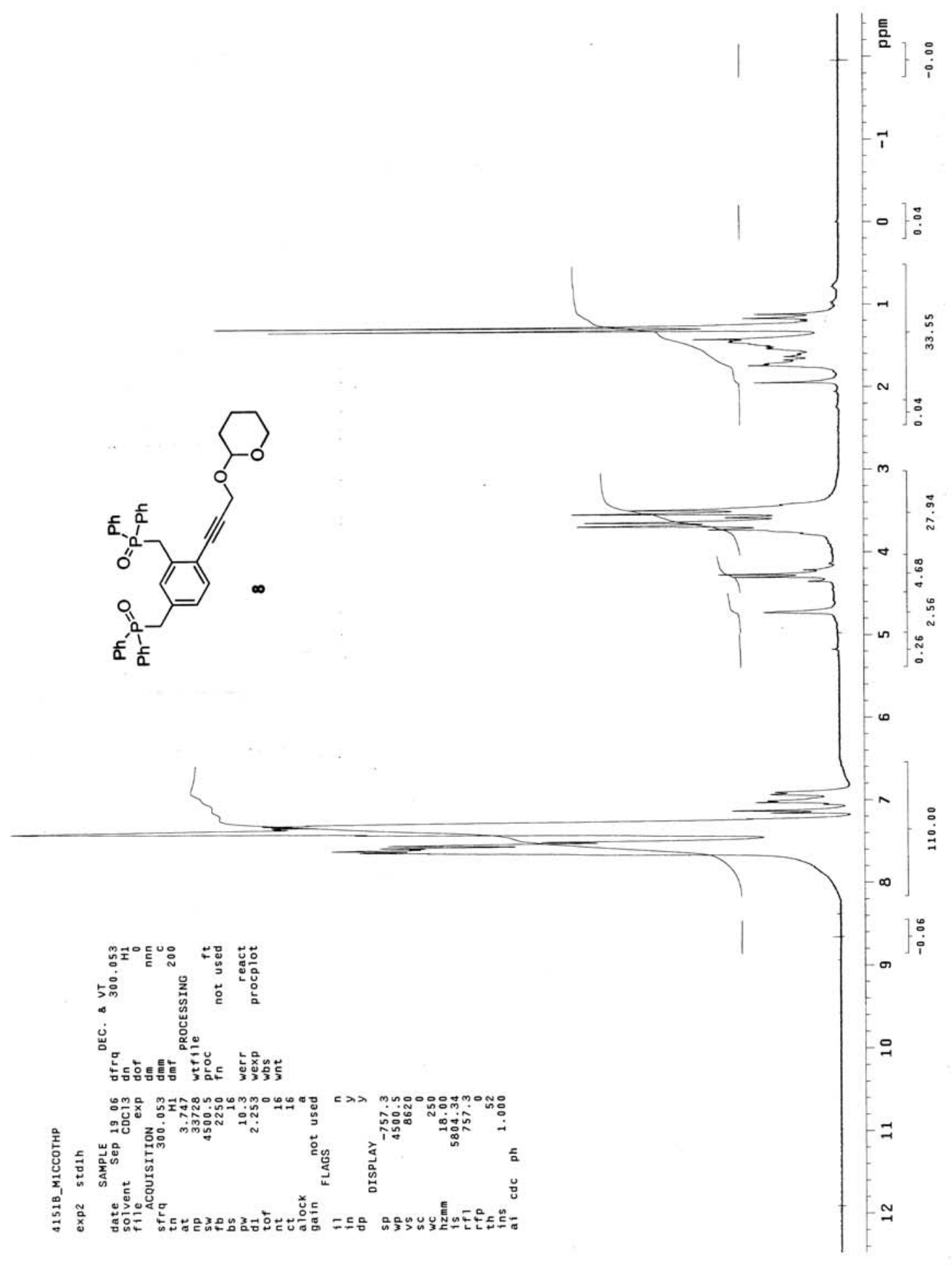




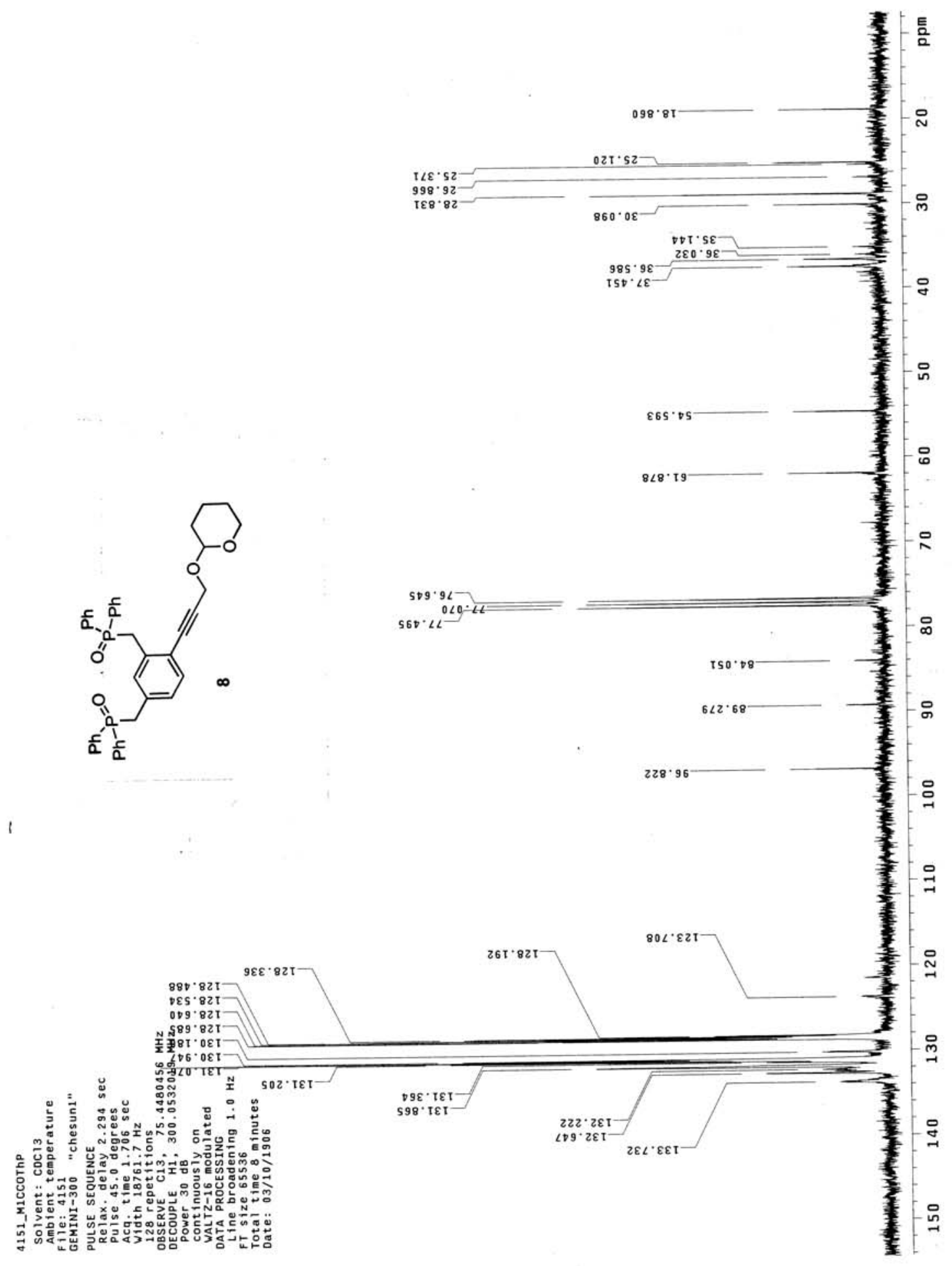




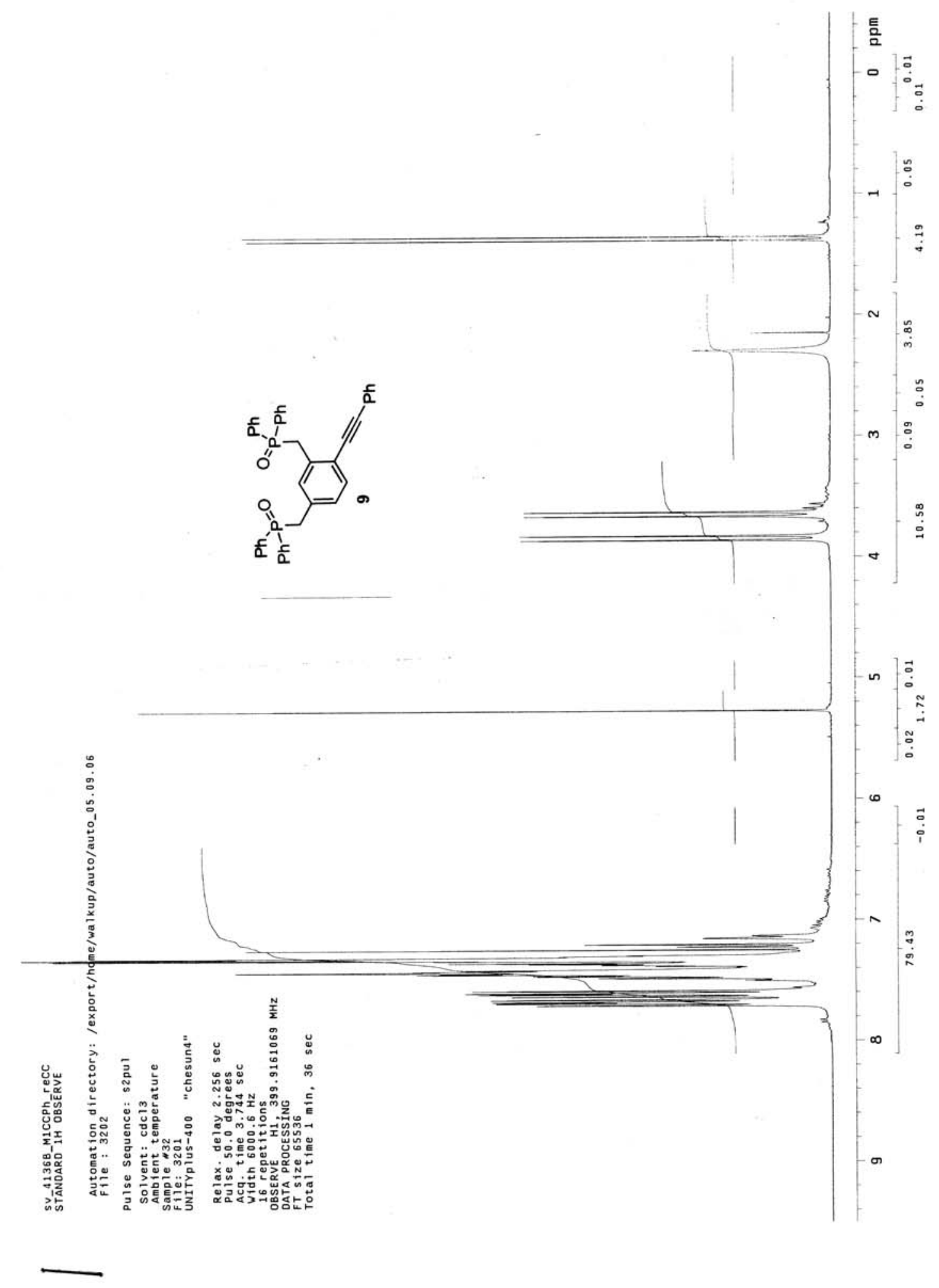



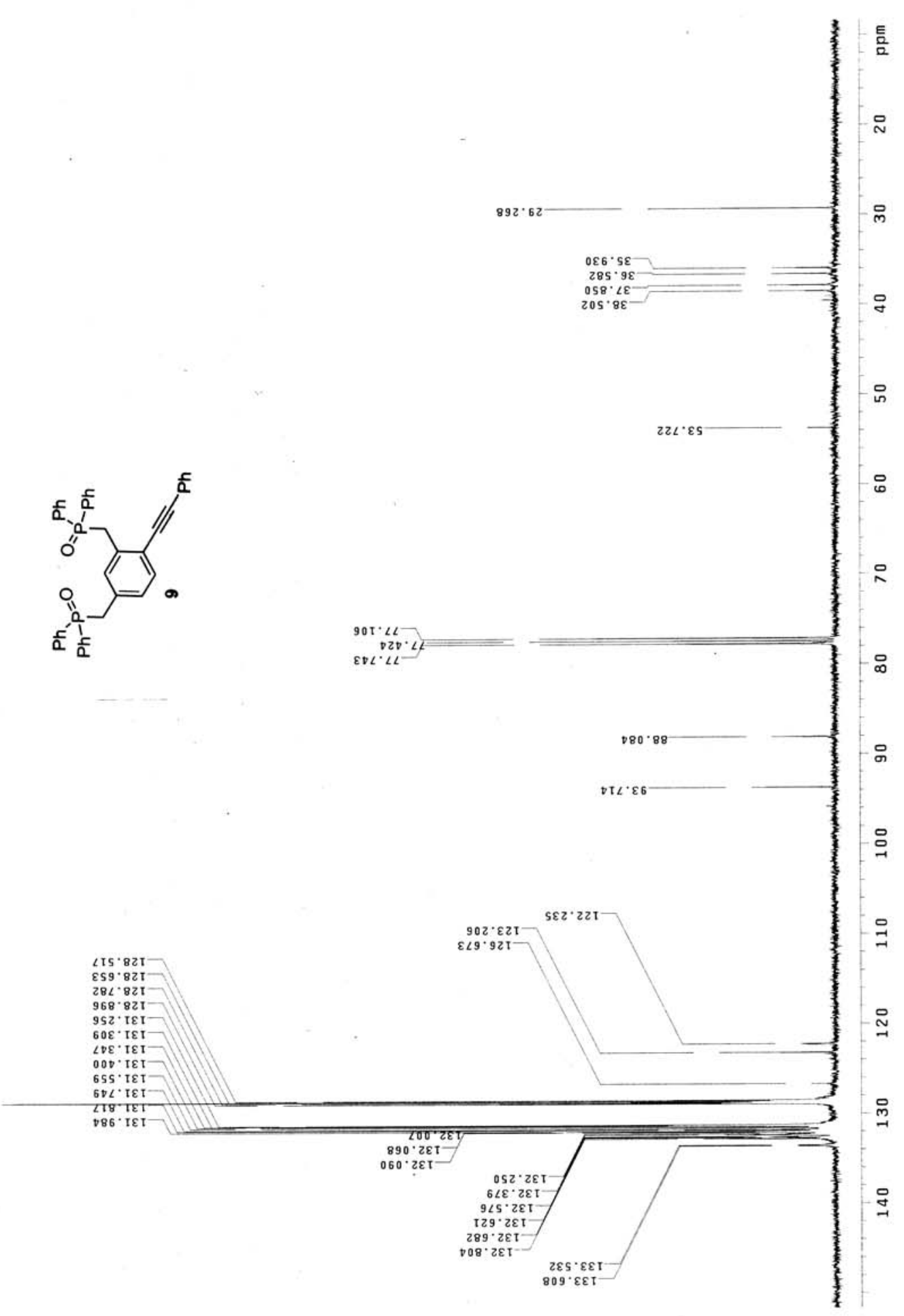


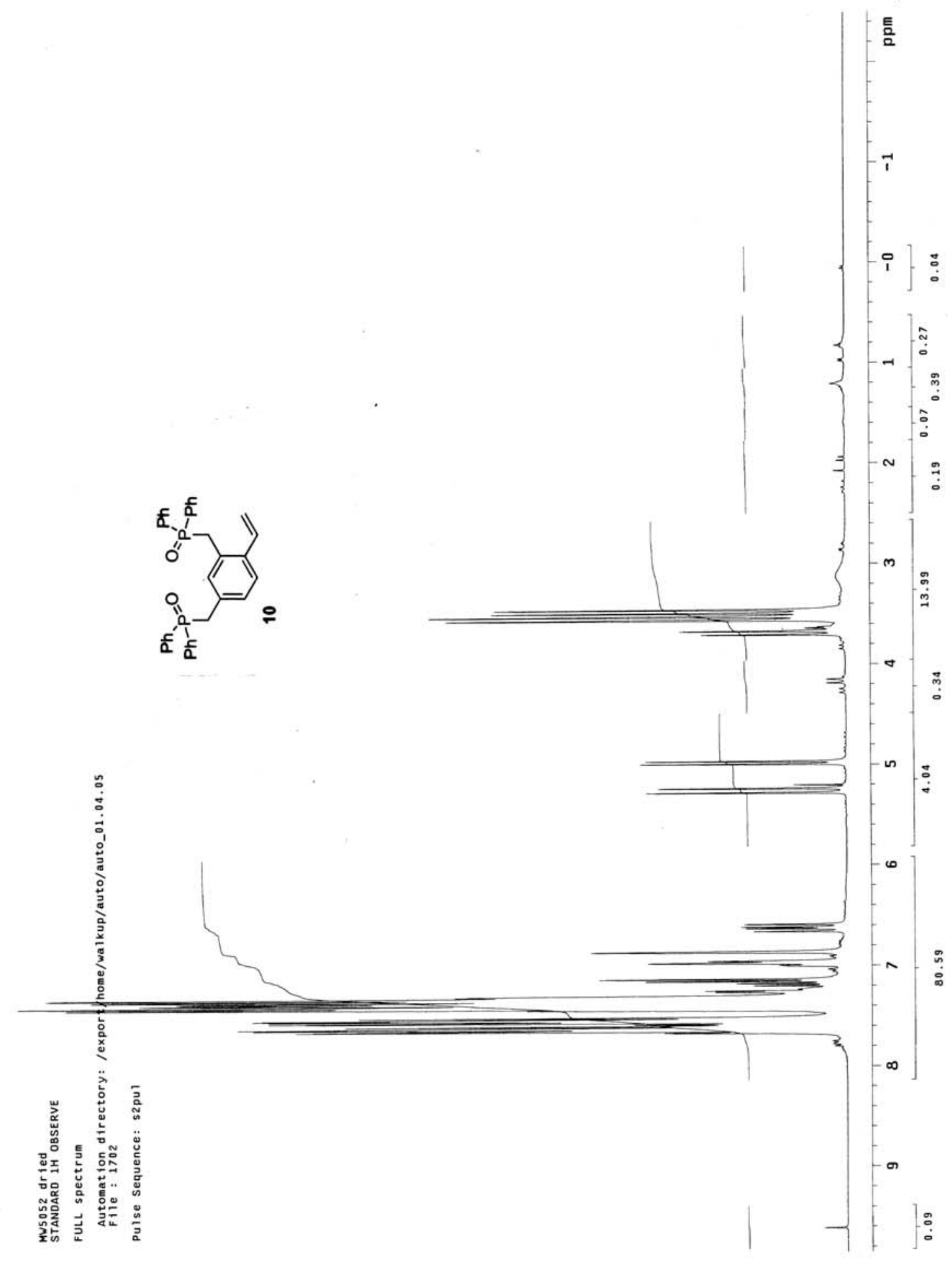




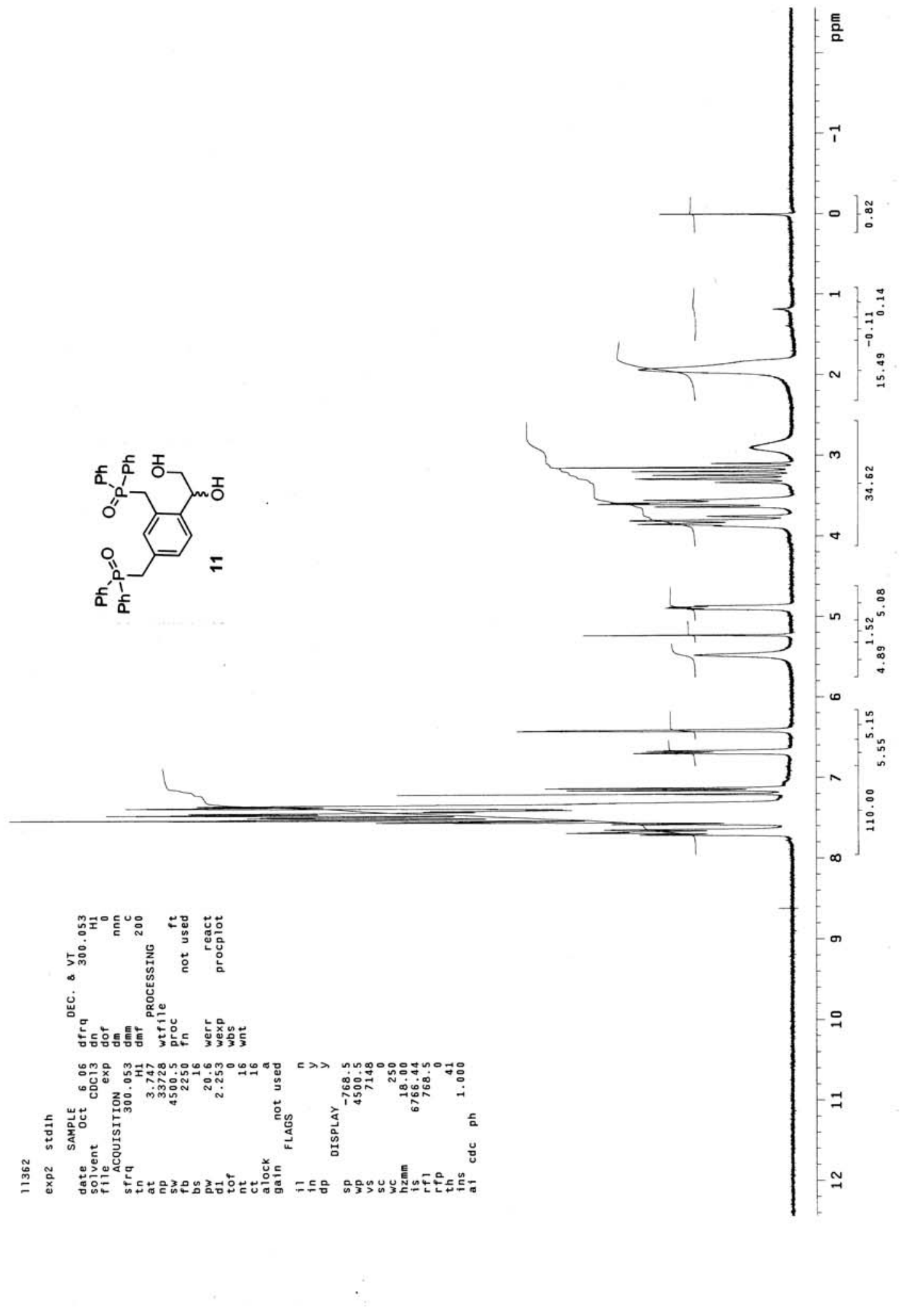




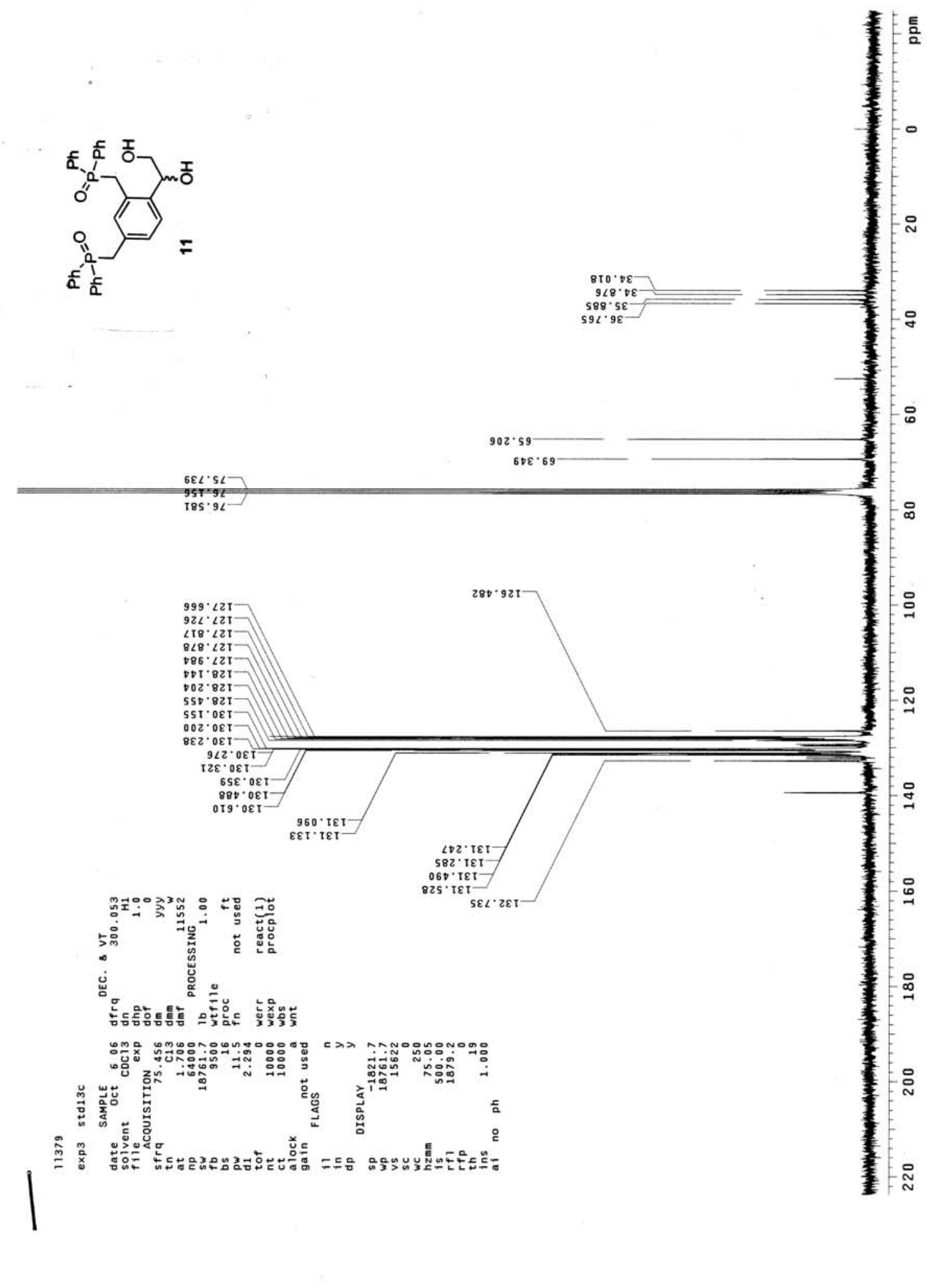




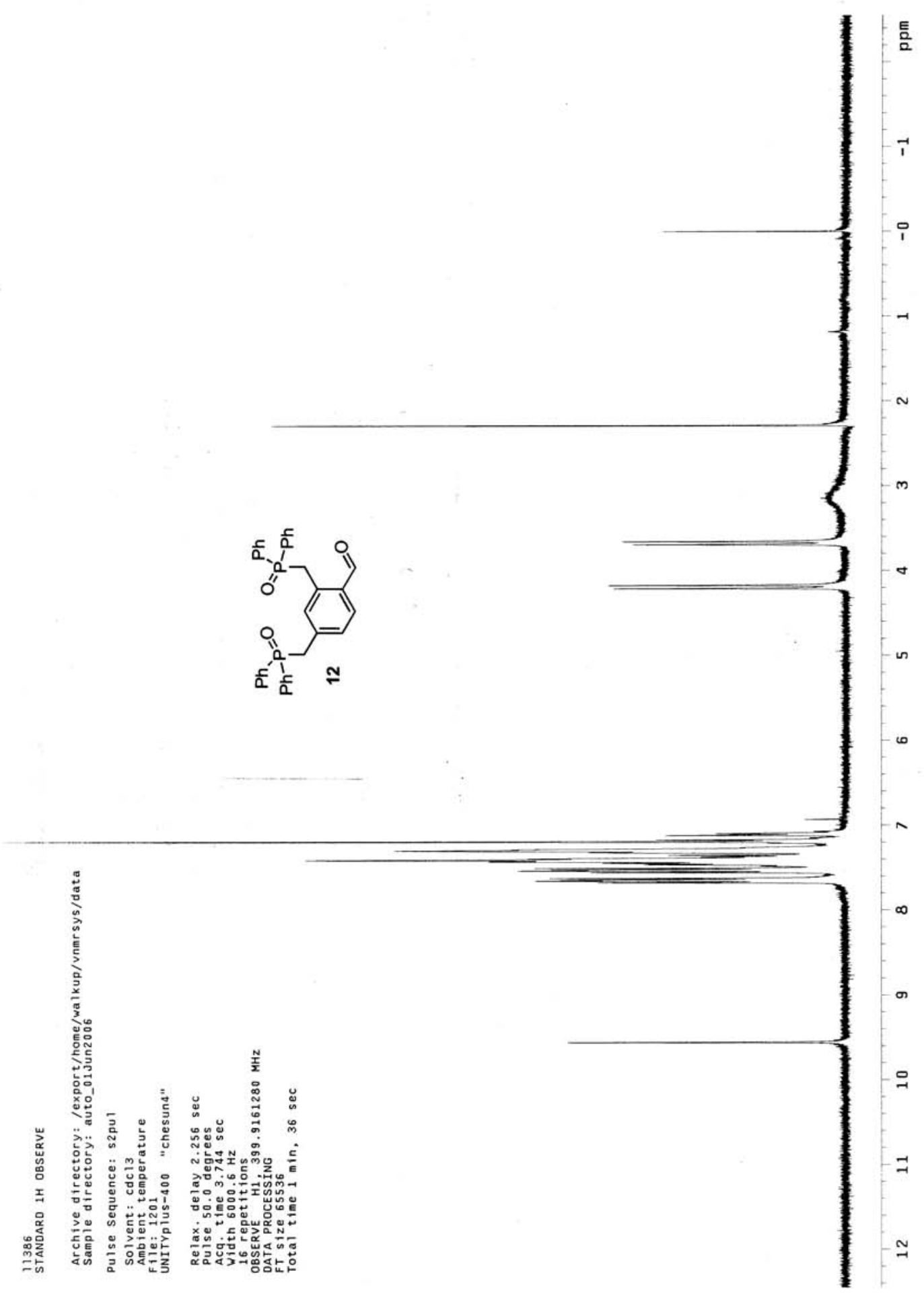



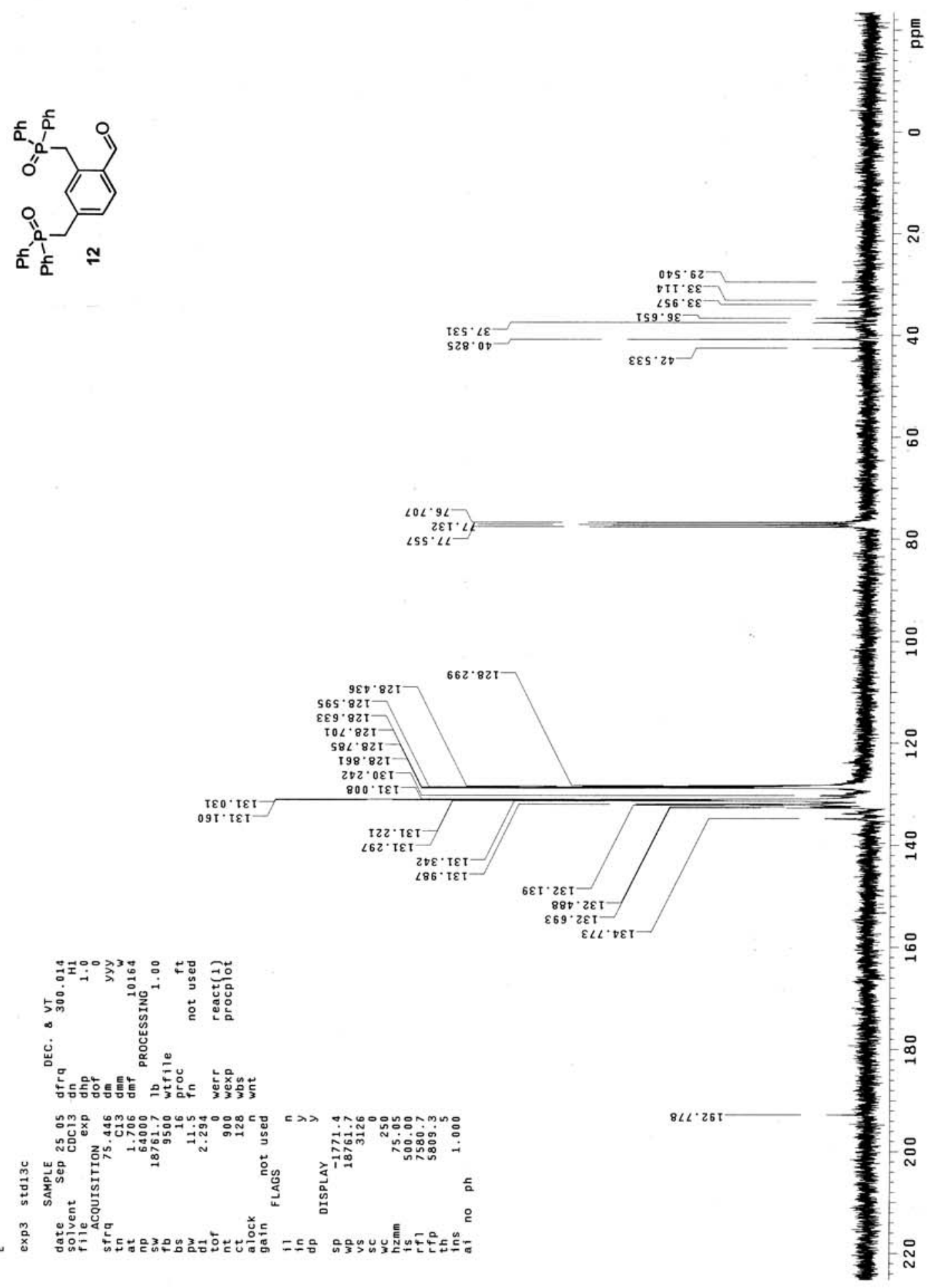


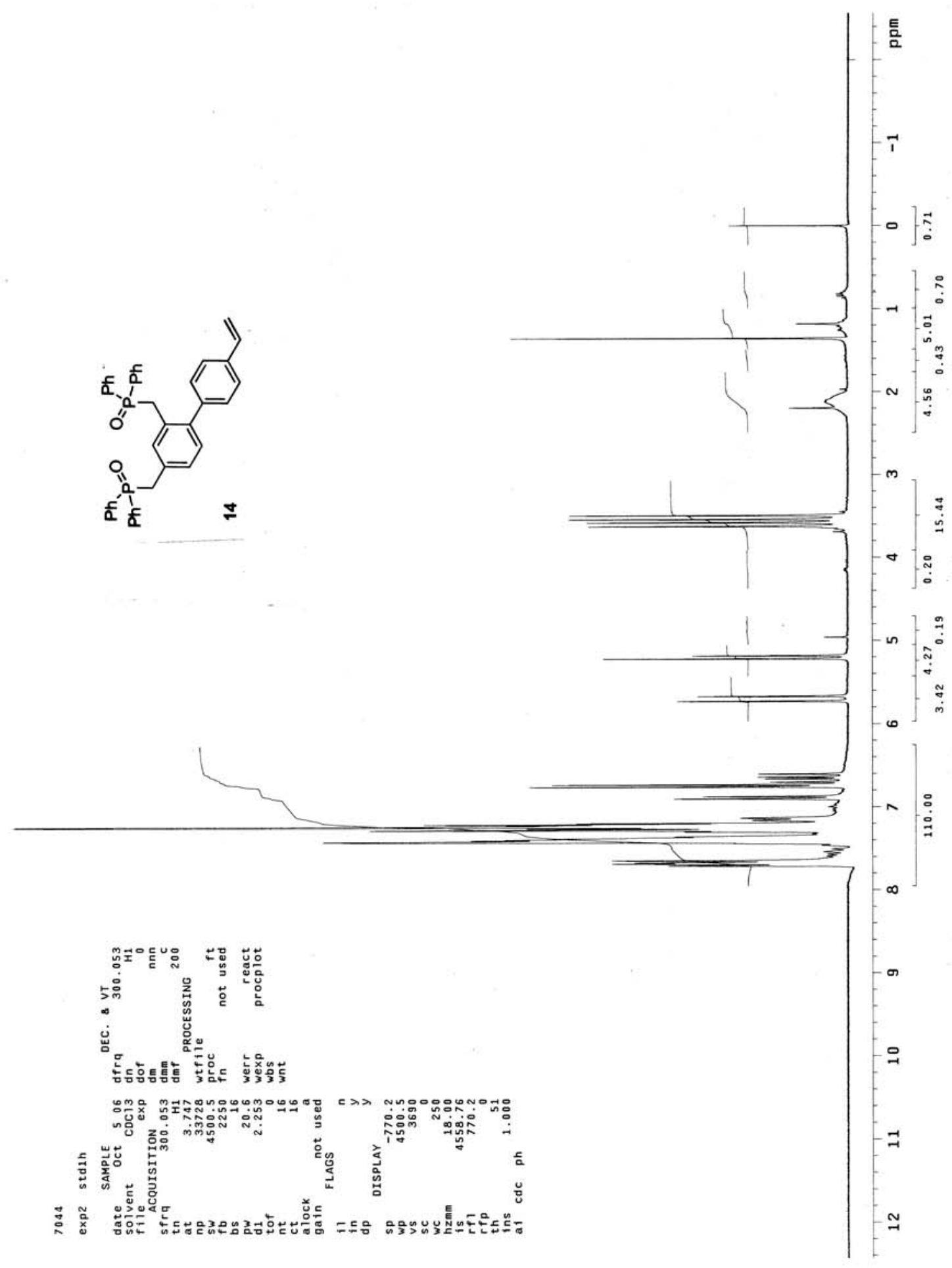



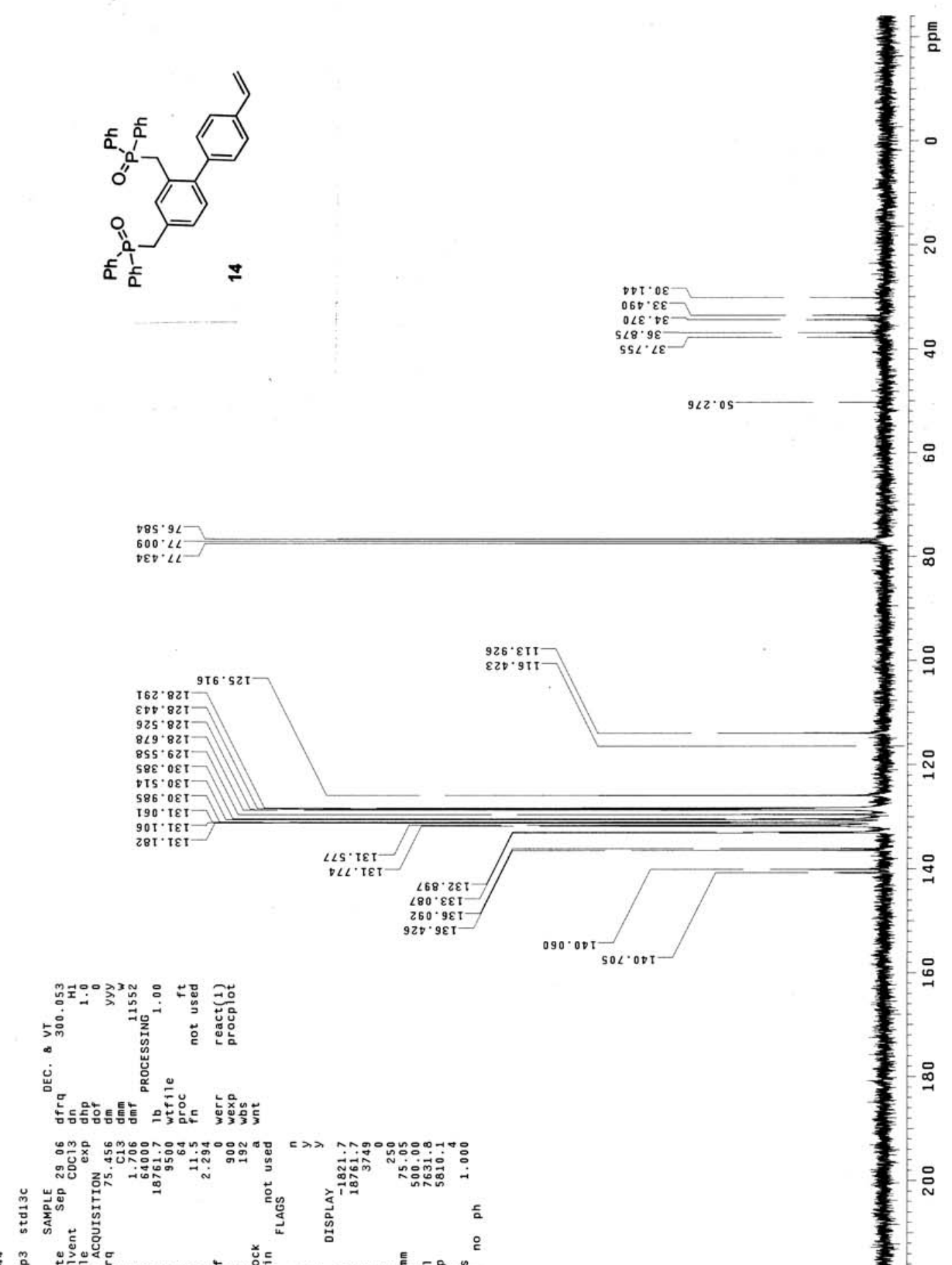

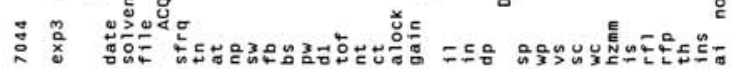




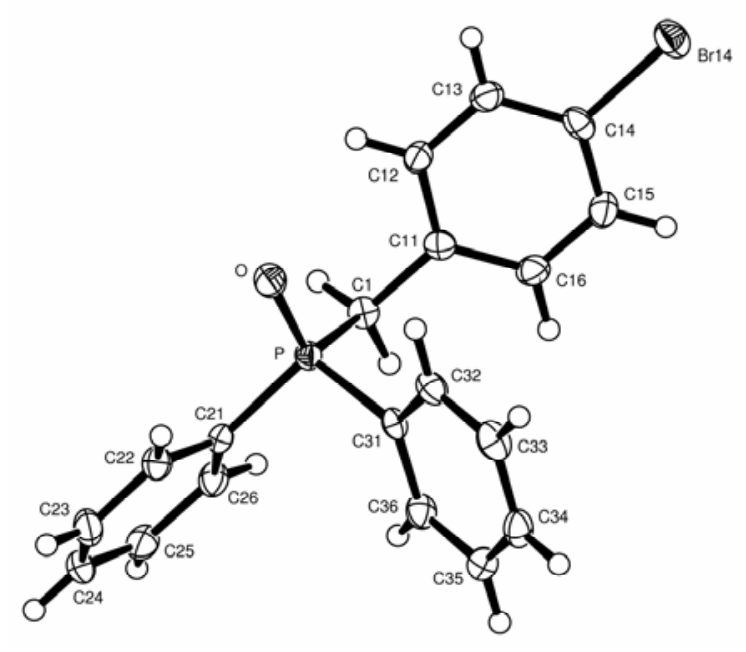

Figure 1. X-ray crystal structure of (diphenylphosphinoylmethyl)benzene 1

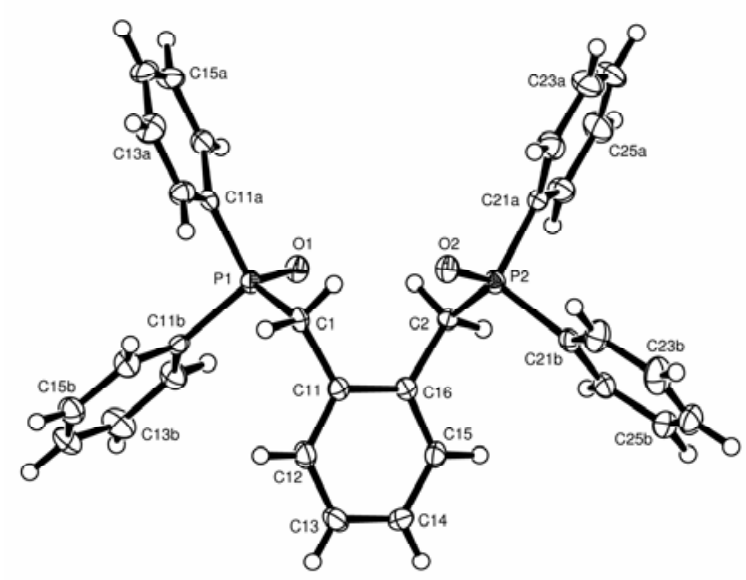

Figure 2. X-ray crystal structure of 1,2-bis(diphenylphosphinonylmethyl)benzene 2

\section{References}

1. Ningning, Z.; Neckers, D. C. J. Org. Chem., 2000, 65, 2145 - 2150

2. Aguiar, A. M.; Nair, M. G. R. J. Org. Chem., 1968, 33, $579-582$

3. J. Chem. Soc., Perkin Trans 1., 1999, $1095-1103$ 